\title{
Continuation of the connection matrix for singularly perturbed hyperbolic equations
}

\author{
by \\ Maria C. Carbinatto (São Carlos) and \\ Krzysztof P. Rybakowski (Rostock)
}

Abstract. Let $\Omega \subset \mathbb{R}^{N}, N \leq 3$, be a bounded domain with smooth boundary, $\gamma \in L^{2}(\Omega)$ be arbitrary and $\phi: \mathbb{R} \rightarrow \mathbb{R}$ be a $C^{1}$-function satisfying a subcritical growth condition. For every $\varepsilon \in] 0, \infty\left[\right.$ consider the semiflow $\pi_{\varepsilon}$ on $H_{0}^{1}(\Omega) \times L^{2}(\Omega)$ generated by the damped wave equation

$$
\begin{aligned}
\varepsilon \partial_{t t} u+\partial_{t} u & =\Delta u+\phi(u)+\gamma(x), & & x \in \Omega, t>0, \\
u(x, t) & =0, & & x \in \partial \Omega, t>0 .
\end{aligned}
$$

Moreover, let $\pi^{\prime}$ be the semiflow on $H_{0}^{1}(\Omega)$ generated by the parabolic equation

$$
\begin{aligned}
\partial_{t} u & =\Delta u+\phi(u)+\gamma(x), & & x \in \Omega, t>0, \\
u(x, t) & =0, & & x \in \partial \Omega, t>0 .
\end{aligned}
$$

Let $\Gamma: H^{2}(\Omega) \rightarrow H_{0}^{1}(\Omega) \times L^{2}(\Omega)$ be the imbedding $u \mapsto(u, \Delta u+\phi(u)+\gamma)$. We prove that whenever $K^{\prime}$ is a compact isolated $\pi^{\prime}$-invariant set and $\left(M_{p}^{\prime}\right)_{p \in P}$ is a partially ordered Morse decomposition of $K^{\prime}$ then the imbedded sets $K=\Gamma\left(K^{\prime}\right)$ and $M_{p, 0}=\Gamma\left(M_{p}^{\prime}\right)$, $p \in P$, continue, for $\varepsilon>0$ small, to an isolated $\pi_{\varepsilon}$-invariant set $K_{\varepsilon}$ a Morse decomposition $\left(M_{p, \varepsilon}\right)_{p \in P}$ of $K_{\varepsilon}$, relative to $\pi_{\varepsilon}$, such that the homology index braid of $\left(\pi_{\varepsilon}, K_{\varepsilon},\left(M_{p, \varepsilon}\right)_{p \in P}\right)$ is isomorphic to the homology index braid of $\left(\pi^{\prime}, K^{\prime},\left(M_{p}^{\prime}\right)_{p \in P}\right)$. This, in particular, implies a connection matrix continuation principle.

1. Introduction. Let $N \in\{1,2,3\}$ and $\Omega \subset \mathbb{R}^{N}$ be a bounded domain with smooth boundary, $\gamma \in L^{2}(\Omega)$ be arbitrary and $\phi: \mathbb{R} \rightarrow \mathbb{R}$ be a $C^{1}$ function such that, for $N \geq 2$, there are constants $\bar{r}$ and $\bar{C} \in[0, \infty[$ with $\left|\phi^{\prime}(u)\right| \leq \bar{C}\left(1+|u|^{\bar{r}}\right)$ for $u \in \mathbb{R}$. If $N=3$ we also assume that $\bar{r}<2$, i.e. that $\phi$ has subcritical growth.

2000 Mathematics Subject Classification: Primary 37B30, 35B25; Secondary 37L05, $37 \mathrm{~L} 65$.

Key words and phrases: parabolic equations, damped hyperbolic equations, connection matrix continuation.

The research of the first author was partially supported by the grants CAPES-Brazil 0710-12/04 and CNPq-Brazil 302854/2002-9. The research of the second author was partially supported by the grant DAAD-Germany D/04/40407. 
For every $\varepsilon \in] 0, \infty[$ consider the following damped wave equation:

$$
\begin{aligned}
\varepsilon \partial_{t t} u+\partial_{t} u & =\Delta u+\phi(u)+\gamma(x), & & x \in \Omega, t>0, \\
u(x, t) & =0 & & x \in \partial \Omega, t>0 .
\end{aligned}
$$

It is well-known that equation $\left(\operatorname{Hyp}_{\varepsilon}\right)$ generates a local semiflow (actually, a local flow) $\pi_{\varepsilon}$ on $H_{0}^{1}(\Omega) \times L^{2}(\Omega)$.

Setting, formally, $\varepsilon=0$ in equation $\left(\mathrm{Hyp}_{\varepsilon}\right)$ we obtain the parabolic equation

$$
\begin{aligned}
\partial_{t} u & =\Delta u+\phi(u)+\gamma(x), & & x \in \Omega, t>0, \\
u(x, t) & =0, & & x \in \partial \Omega, t>0 .
\end{aligned}
$$

Again it is well-known that equation (Par) generates a local semiflow $\pi^{\prime}$ on $H_{0}^{1}(\Omega)$.

It is a natural question whether, for $\varepsilon \rightarrow 0$, solutions of $\pi_{\varepsilon}$ converge, in some sense, to solutions of $\pi^{\prime}$, properly imbedded into $H_{0}^{1}(\Omega) \times L^{2}(\Omega)$. This question was considered in the context of attractors by Hale and Raugel [12] and in the context of Conley index theory by the second author in [17].

In fact, in [17] the following result was proved.

Theorem A. Let $\Gamma: H^{2}(\Omega) \rightarrow H_{0}^{1}(\Omega) \times L^{2}(\Omega)$ be the imbedding $u \mapsto$ $(u, \Delta u+\phi(u)+\gamma)$. Moreover, let $K^{\prime}$ be a compact $\left(\right.$ in $\left.H_{0}^{1}(\Omega)\right)$ isolated invariant set relative to $\pi^{\prime}$. Then $K^{\prime} \subset H^{2}(\Omega)$, and the set $K_{0}:=\Gamma\left(K^{\prime}\right)$ continues to a family $\left(K_{\varepsilon}\right)_{\varepsilon \in\left[0, \widetilde{\varepsilon}_{0}\right]}$, where $\widetilde{\varepsilon}_{0}>0$, such that for every $\left.\left.\varepsilon \in\right] 0, \widetilde{\varepsilon}_{0}\right], K_{\varepsilon}$ is a compact isolated invariant set relative to $\pi_{\varepsilon}$ such that the Conley index of $\left(\pi_{\varepsilon}, K_{\varepsilon}\right)$ is equal to the Conley index of $\left(\pi^{\prime}, K^{\prime}\right)$. Furthermore, $\left(K_{\varepsilon}\right)_{\varepsilon \in\left[0, \widetilde{\varepsilon}_{0}\right]}$ is upper-semicontinuous at $\varepsilon=0$.

Moreover, let $\left(M_{p}^{\prime}\right)_{p \in P}$ be $a \prec$-ordered Morse decomposition of $K^{\prime}$ relative to $\pi^{\prime}$. Then the family $\left(M_{p, 0}\right)_{p \in P}:=\left(\Gamma\left(M_{p}^{\prime}\right)\right)_{p \in P}$ continues to a family $\left(\left(M_{p, \varepsilon}\right)_{p \in P}\right)_{\varepsilon \in\left[0, \widetilde{\varepsilon}_{0}\right]}$, where for every $\left.\left.\varepsilon \in\right] 0, \widetilde{\varepsilon}_{0}\right],\left(M_{p, \varepsilon}\right)_{p \in P}$ is a $\prec$-ordered Morse decomposition of $K_{\varepsilon}$ relative to $\pi_{\varepsilon}$. Furthermore, $\left(\left(M_{p, \varepsilon}\right)_{p \in P}\right)_{\varepsilon \in\left[0, \widetilde{\varepsilon}_{0}\right]}$ is upper-semicontinuous at $\varepsilon=0$.

We refer to [17, Theorems 6.1 and 7.4, and Remark 7.5] for the precise statement of a more general result.

Theorem A extends previous upper-semicontinuity results for attractors by Hale and Raugel.

The main goal of this paper is to refine Theorem A to a continuation result for homology index braids. More precisely, we shall prove

Theorem B. In the notation of Theorem A, for all $\left.\varepsilon \in] 0, \varepsilon_{0}\right]$, the (co)homology index braids of $\left(\pi^{\prime}, K^{\prime},\left(M_{p}^{\prime}\right)_{p \in P}\right)$ and $\left(\pi_{\varepsilon}, K_{\varepsilon},\left(M_{p, \varepsilon}\right)_{p \in P}\right)$ are isomorphic.

We recall that if two homology index braids $\mathcal{H}_{1}$ and $\mathcal{H}_{2}$ are isomorphic, then, by [10, Proposition 1.5], $\mathcal{H}_{1}$ and $\mathcal{H}_{2}$ determine the same collection 
of $C$-connection matrices. Thus Theorem B shows that, for small $\varepsilon>0$, the Morse decomposition $\left(M_{p, \varepsilon}\right)_{p \in P}$ of the invariant set $K_{\varepsilon}$ relative to the damped wave equation $\left(\mathrm{Hyp}_{\varepsilon}\right)$ has the same set of $C$-connection matrices as the Morse decomposition $\left(M_{p}^{\prime}\right)_{p \in P}$ of the invariant set $K^{\prime}$ relative to the reaction-diffusion equation (Par). In particular, this means that homologically nontrivial heteroclinic connections of the simpler equation (Par) survive in the more complicated equation $\left(\operatorname{Hyp}_{\varepsilon}\right)$.

Theorem B will be proved by an application of some abstract continuation results for regularly and singularly perturbed semiflows, recently established in our papers [5] and [7]. Our approach to the proof of Theorem B is similar to the proof of Theorem A in [17]. As in [17], the first attempt would be to make a change of variables

$$
\Phi:(u, v) \mapsto(u, w):=(u, v-\Delta u-\phi(u)-\gamma)
$$

in $\left(\operatorname{Hyp}_{\varepsilon}\right)$, consider the corresponding conjugate semiflows $\widetilde{\pi}_{\varepsilon}=\Phi^{*} \pi_{\varepsilon}, \varepsilon \in$ ] $0, \infty\left[\right.$, and then apply the results of [7] to the family $\widetilde{\pi}_{\varepsilon}, \varepsilon \in[0, \infty[$, where $\widetilde{\pi}_{0}=\pi^{\prime}$. However, as it was pointed out in [17], there is an inherent difficulty in the present situation due to the fact that the transformation $\Phi$ is defined on the space $H^{2}(\Omega) \times L^{2}(\Omega)$, which is only a subset of the phase space $H_{0}^{1}(\Omega) \times L^{2}(\Omega)$ of the semiflows $\pi_{\varepsilon}$, so $\widetilde{\pi}_{\varepsilon}$ is not well-defined for $\left.\varepsilon \in\right] 0, \infty[$. That is why we first study, in Section 3, a finite-dimensional perturbation problem (equations (3.1) and (3.2) below), to which a variable transformation like $\Phi$ is applicable. Results from [7] then yield a continuation result for homology index braids for the corresponding family of finite-dimensional semiflows (cf. Theorems 3.1 and 3.6 below).

Combining the compactness and smoothing results for parabolic equations and the boundedness and smoothing results for damped wave equations established in [17] with the results from Section 3, we then obtain, in the last section of the paper, our main result, Theorem 4.2, which implies Theorem B above.

2. Preliminaries. The purpose of this section is to recall a few concepts from Conley index theory and some preliminary results needed later in this paper. We assume the reader's familiarity with the infinite-dimensional Conley index theory, as developed in [14], [15] and expounded in [16], and with the papers [9], [11], [5] and [6]. Moreover, we recall some results from the papers [1], [4], [7] and [17].

In this section, unless otherwise specified, $Y$ is a metric space, $\pi$ is a local semiflow on $Y$ and all concepts are defined relative to $\pi$.

Suppose that $Z$ is a subset of $Y$. We denote by $\operatorname{Inv}_{\pi}(Z)$ the largest invariant subset of $Z$. 
$Z$ is called $\pi$-admissible if it is closed and whenever $\left(x_{n}\right)_{n}$ and $\left(t_{n}\right)_{n}$ are such that $t_{n} \rightarrow \infty$ and $x_{n} \pi\left[0, t_{n}\right] \subset Z$ for all $n \in \mathbb{N}$, then the sequence $\left(x_{n} \pi t_{n}\right)_{n}$ has a convergent subsequence. We say that $\pi$ does not explode in $Z$ if whenever $x \in Y$ and $x \pi t \in Z$ as long as $x \pi t$ is defined, then $x \pi t$ is defined for all $t \in[0, \infty[$. Moreover $Z$ is called strongly $\pi$-admissible if $Z$ is $\pi$-admissible and $\pi$ does not explode in $Z$.

Let $N$ and $Z$ be subsets of $Y$. The set $Z$ is called $N$-positively invariant if whenever $x \in Z, t \geq 0$ are such that $x \pi[0, t] \subset N$, then $x \pi[0, t] \subset Z$.

Let $N, Z_{1}$ and $Z_{2}$ be subsets of $Y$. Then $Z_{2}$ is called an exit ramp for $N$ within $Z_{1}$ if whenever $x \in Z_{1}$ and $x \pi t^{\prime} \notin N$ for some $t^{\prime} \in[0, \infty$ [, then there exists a $t_{0} \in\left[0, t^{\prime}\right]$ such that $x \pi\left[0, t_{0}\right] \subset N$ and $x \pi t_{0} \in Z_{2}$.

If $Z_{1}$ and $Z_{2}$ are subsets of $Y$ then $Z_{2}$ is called an exit ramp for $Z_{1}$ if $Z_{2}$ is an exit ramp for $N$ within $Z_{1}$, where $N=Z_{1}$.

Definition 2.1 ([11]). A pair $\left(N_{1}, N_{2}\right)$ is called a Franzosa-Mischaikowindex pair (or FM-index pair) for $S$ if:

(1) $N_{1}$ and $N_{2}$ are closed subsets of $Y$ with $N_{2} \subset N_{1}$ and $N_{2}$ is $N_{1^{-}}$ positively invariant;

(2) $N_{2}$ is an exit ramp for $N_{1}$;

(3) $S$ is closed, $S \subset \operatorname{Int}_{Y}\left(N_{1} \backslash N_{2}\right)$ and $S$ is the largest invariant set in $\mathrm{Cl}_{Y}\left(N_{1} \backslash N_{2}\right)$.

Given an isolated invariant set $K$ having a strongly $\pi$-admissible isolating neighborhood we denote by $h(K)=h(\pi, K)$ the Conley index of $K$ and by $H(K)=H(\pi, K)=H(h(K))$ the homology Conley index, where $H$ is the singular homology functor (with coefficients in some fixed $R$-module $G$, where $R$ is a commutative ring).

For the rest of this paper let $P$ be a fixed finite set and $\prec$ be a fixed strict partial order on $P$.

A set $I \subset P$ is called a $\prec$-interval if whenever $i, j, k \in P, i, k \in I$ and $i \prec j \prec k$, then $j \in I$. We denote by $\mathcal{I}(\prec)$ the set of all $\prec$-intervals in $P$. The set $I$ is called a $\prec$-attracting interval if whenever $i, j \in P, j \in I$ and $i \prec j$, then $i \in I$. Let $\mathcal{A}(\prec)$ denote the set of all $\prec$-attracting intervals in $P$. Of course, $\mathcal{A}(\prec) \subset \mathcal{I}(\prec)$.

An adjacent $n$-tuple of $\prec$-intervals is a sequence $\left(I_{j}\right)_{j=1}^{n}$ of pairwise disjoint $\prec$-intervals whose union is a $\prec$-interval and such that, whenever $j<k$, $p \in I_{j}$ and $p^{\prime} \in I_{k}$, then $p^{\prime} \nprec p$ (i.e. $p \prec p^{\prime}$ or else $p$ and $p^{\prime}$ are not related by $\prec)$. We denote by $\mathcal{I}_{n}(\prec)$ the set of all adjacent $n$-tuples of $\prec$-intervals.

Recall the following definition.

Definition 2.2 (cf. [11]). Let $\pi$ be a local semiflow on $Y$ and $S$ be a compact invariant set relative to $\pi$. A family $\left(M_{p}\right)_{p \in P}$ of subsets of $S$ is called a $\prec$-ordered Morse decomposition of $S$ (relative to $\pi$ ) if the following 
properties hold:

(1) The sets $M_{p}, p \in P$, are closed, $\pi$-invariant and pairwise disjoint.

(2) For every full solution $\sigma$ of $\pi$ lying in $S$, either $\sigma(\mathbb{R}) \subset M_{k}$ for some $k \in P$, or there are $k, l \in P$ with $k \prec l, \alpha(\sigma) \subset M_{l}$ and $\omega(\sigma) \subset M_{k}$.

Let $\mathcal{C}$ denote the set of all continuous functions from $\mathbb{R}$ to $Y$ endowed with the metric introduced in [1]. Let $\mathcal{T}$ be a subset of $\mathcal{C}$. A set $S \subset Y$ is called $\mathcal{T}$-invariant if $S=\operatorname{Inv}_{\mathcal{T}}(S)$, i.e. if and only if for every $y \in S$ there is a $\sigma \in \mathcal{T}$ such that $\sigma(\mathbb{R}) \subset S$ and $y=\sigma(0)$. If $\pi$ is a local semiflow on $Y$ and $N \subset Y$ then we denote by $\mathcal{T}_{\pi, N}$ the set of all full solutions of $\pi$ lying in $N$.

The previous concept can be generalized as follows:

Definition 2.3 (cf. [4]). Let $\mathcal{T}$ be a subset of $\mathcal{C}$. A family $\left(M_{p}\right)_{p \in P}$ of subsets of $Y$ is called a $\prec$-ordered $\mathcal{T}$-Morse decomposition if the following properties hold:

(1) The sets $M_{p}, p \in P$, are closed, $\mathcal{T}$-invariant and pairwise disjoint.

(2) For every $\sigma \in \mathcal{T}$ either $\sigma(\mathbb{R}) \subset M_{k}$ for some $k \in P$ or else there are $k, l \in P$ with $k \prec l, \alpha(\sigma) \subset M_{l}$ and $\omega(\sigma) \subset M_{k}$.

It is easily proved that, for $\pi$ and $S$ as in Definition 2.2 , a family $\left(M_{p}\right)_{p \in P}$ of subsets of $S$ is a $\prec$-ordered Morse decomposition of $S$ (relative to $\pi$ ) if and only if $\left(M_{p}\right)_{p \in P}$ is a $\prec$-ordered $\mathcal{T}$-Morse decomposition, where $\mathcal{T}:=\mathcal{T}_{\pi, S}$.

If $A, B \subset Y$ then the $\mathcal{T}$-connection set $\operatorname{CS}_{\mathcal{T}}(A, B)$ from $A$ to $B$ is the set of all points $y \in Y$ for which there is a $\sigma \in \mathcal{T}$ with $\sigma(0)=y, \alpha(\sigma) \subset A$ and $\omega(\sigma) \subset B$. If $\pi, S$ are as in Definition 2.2 and $\mathcal{T}:=\mathcal{T}_{\pi, S}$, then we write

$$
\mathrm{CS}_{\pi, S}(A, B):=\mathrm{CS}_{\mathcal{T}}(A, B) \text {. }
$$

Recall also the following definitions.

DEFINITION 2.4 (cf. [17]). We say that $N$ is a $\mathcal{T}$-isolating neighborhood (of a subset $S$ of $Y$ ) if $N$ is closed in $Y$ and $\operatorname{Inv}_{\mathcal{T}}(N) \subset \operatorname{Int}_{Y}(N)$ (with $\left.S=\operatorname{Inv}_{\mathcal{T}}(N)\right)$. If $S \subset Y$ and there exists a set $N \subset Y$ such that $N$ is a $\mathcal{T}$-isolating neighborhood of $S$ then we call $S$ a $\mathcal{T}$-isolated invariant set.

Definition 2.5 (cf. [1]). Given $\sigma_{1}$ and $\sigma_{2}$ in $\mathcal{C}$ with $\sigma_{1}(0)=\sigma_{2}(0)$ the $\operatorname{map} \sigma_{1} \triangleright \sigma_{2}: \mathbb{R} \rightarrow X$ defined by

$$
\left(\sigma_{1} \triangleright \sigma_{2}\right)(t)= \begin{cases}\sigma_{1}(t) & \text { if } t \leq 0, \\ \sigma_{2}(t) & \text { if } t \geq 0,\end{cases}
$$

is called the cut-and-glue of $\left(\sigma_{1}, \sigma_{2}\right)$. A subset $\mathcal{T}$ of $\mathcal{C}$ is called cut-and-glueinvariant if $\sigma_{1} \triangleright \sigma_{2} \in \mathcal{T}$ for all $\sigma_{1}, \sigma_{2} \in \mathcal{T}$ with $\sigma_{1}(0)=\sigma_{2}(0)$.

For the rest of this paper, unless specified otherwise, let $\left(X_{0}, d_{0}\right)$ be a metric space, $\varepsilon_{0}$ be a positive number and, for each $\left.\left.\varepsilon \in\right] 0, \varepsilon_{0}\right],\left(Y_{\varepsilon}, d_{\varepsilon}\right)$ be a metric space and $\theta_{\varepsilon} \in Y_{\varepsilon}$ be a distinguished point of $Y_{\varepsilon}$. The open ball in $Y_{\varepsilon}$ with center $v$ and radius $\beta>0$ is denoted by $B_{\varepsilon}(v, \beta)$. 
For each $\left.\varepsilon \in] 0, \varepsilon_{0}\right]$ define the set $Z_{\varepsilon}:=X_{0} \times Y_{\varepsilon}$. Endow $Z_{\varepsilon}$ with the metric

$$
\Gamma_{\varepsilon}\left((u, v),\left(u^{\prime}, v^{\prime}\right)\right):=\max \left\{d_{0}\left(u, u^{\prime}\right), d_{\varepsilon}\left(v, v^{\prime}\right)\right\}, \quad(u, v),\left(u^{\prime}, v^{\prime}\right) \in Z_{\varepsilon} .
$$

Given a subset $V$ of $X_{0}, \beta>0$ and $\left.\varepsilon \in\right] 0, \varepsilon_{0}$ ] define the "inflated" subset $[V]_{\varepsilon, \beta}$ of $Z_{\varepsilon}$ as follows:

$$
[V]_{\varepsilon, \beta}:=\left\{(u, v) \in Z_{\varepsilon} \mid u \in V \text { and } v \in \mathrm{Cl}_{\varepsilon} B_{\varepsilon}\left(\theta_{\varepsilon}, \beta\right)\right\} .
$$

Let $\pi_{0}$ be a local semiflow on $X_{0}$ and for every $\left.\varepsilon \in\right] 0, \varepsilon_{0}$ ] let $\pi_{\varepsilon}$ denote a local semiflow on $Z_{\varepsilon}$. We say that the family $\left(\pi_{\varepsilon}\right)_{\left.\varepsilon \in] 0, \varepsilon_{0}\right]}$ of local semiflows converges singularly to the local semiflow $\pi_{0}$ if whenever $\left(\varepsilon_{n}\right)_{n}$ and $\left(t_{n}\right)_{n}$ are sequences of positive (resp. nonnegative) numbers such that $\varepsilon_{n} \rightarrow 0, t_{n} \rightarrow t_{0}$ as $n \rightarrow \infty$, for some $t_{0} \in\left[0, \infty\left[\right.\right.$, and whenever $u_{0} \in X_{0}$ and $w_{n} \in Z_{\varepsilon_{n}}, n \in \mathbb{N}$, are such that $\Gamma_{\varepsilon_{n}}\left(w_{n},\left(u_{0}, \theta_{\varepsilon_{n}}\right)\right) \rightarrow 0$ as $n \rightarrow \infty$ and $u_{0} \pi_{0} t_{0}$ is defined, then there exists an $n_{0} \in \mathbb{N}$ such that for all $n \geq n_{0}, w_{n} \pi_{\varepsilon_{n}} t_{n}$ is defined and $\Gamma_{\varepsilon_{n}}\left(w_{n} \pi_{\varepsilon_{n}} t_{n},\left(u_{0} \pi_{0} t_{0}, \theta_{\varepsilon_{n}}\right)\right) \rightarrow 0$ as $n \rightarrow \infty$.

Let $\eta$ be a positive number and $N$ be a closed subset of $X_{0}$. We say that $N$ is a singularly strongly admissible set with respect to $\eta$ and the family $\left(\pi_{\varepsilon}\right)_{\varepsilon \in\left[0, \varepsilon_{0}\right]}$ of local semiflows if the following conditions are satisfied:

(1) $N$ is a strongly $\pi_{0}$-admissible set;

(2) for each $\left.\varepsilon \in] 0, \varepsilon_{0}\right]$ the set $[N]_{\varepsilon, \eta}$ is strongly $\pi_{\varepsilon}$-admissible;

(3) whenever $\left(\varepsilon_{n}\right)_{n}$ and $\left(t_{n}\right)_{n}$ are sequences of positive numbers such that $\varepsilon_{n} \rightarrow 0, t_{n} \rightarrow \infty$ as $n \rightarrow \infty$ and whenever $w_{n} \in Z_{\varepsilon_{n}}, n \in \mathbb{N}$, are such that $w_{n} \pi_{\varepsilon_{n}}\left[0, t_{n}\right] \subset[N]_{\varepsilon_{n}, \eta}, n \in \mathbb{N}$, then there exist a $u_{0} \in N$ and a subsequence of the sequence $\left(w_{n} \pi_{\varepsilon_{n}} t_{n}\right)_{n}$ of endpoints, denoted again by $\left(w_{n} \pi_{\varepsilon_{n}} t_{n}\right)_{n}$, such that $\Gamma_{\varepsilon_{n}}\left(w_{n} \pi_{\varepsilon_{n}} t_{n},\left(u_{0}, \theta_{\varepsilon_{n}}\right)\right) \rightarrow 0$ as $n \rightarrow \infty$.

The following singular continuation result for Morse decompositions was established in [4].

Theorem 2.6 ([4, Corollaries 4.14 and 4.15]). Assume $\left(\pi_{\varepsilon}\right)_{\left.\varepsilon \in] 0, \varepsilon_{0}\right]}$ is a family of local semiflows that converges singularly to the local semiflow $\pi_{0}$, $\beta \in] 0, \infty[$ and $\widetilde{N}$ is a singularly strongly admissible set with respect to $\beta$ and $\left(\pi_{\varepsilon}\right)_{\varepsilon \in\left[0, \varepsilon_{0}\right]}$. Moreover, suppose that $S_{0}:=\operatorname{Inv}_{\pi_{0}}(\widetilde{N})$ and $\left(M_{p, 0}\right)_{p \in P}$ is $a \prec$-ordered Morse decomposition of $S_{0}$ relative to $\pi_{0}$. For each $p \in P$, let $V_{p} \subset \widetilde{N}$ be closed in $X_{0}$ and such that $M_{p, 0}=\operatorname{Inv}_{\pi_{0}}\left(V_{p}\right) \subset \operatorname{Int}_{X_{0}}\left(V_{p}\right)$.

Let $\eta \in] 0, \beta]$. For $\left.\varepsilon \in] 0, \varepsilon_{0}\right]$ and $p \in P$ set $S_{\varepsilon}:=\operatorname{Inv}_{\pi_{\varepsilon}}\left([\widetilde{N}]_{\varepsilon, \eta}\right)$ and $M_{p, \varepsilon}:=\operatorname{Inv}_{\pi_{\varepsilon}}\left(\left[V_{p}\right]_{\varepsilon, \eta}\right)$. Then there is an $\left.\left.\widetilde{\varepsilon} \in\right] 0, \varepsilon_{0}\right]$ such that for every $\varepsilon \in$ ] $0, \widetilde{\varepsilon}]$ and $p \in P, S_{\varepsilon} \subset \operatorname{Int}_{Z_{\varepsilon}}\left([\tilde{N}]_{\varepsilon, \eta}\right), M_{p, \varepsilon} \subset \operatorname{Int}_{Z_{\varepsilon}}\left(\left[V_{p}\right]_{\varepsilon, \eta}\right), p \in P$, and the family $\left(M_{p, \varepsilon}\right)_{p \in P}$ is a $\prec$-ordered Morse decomposition of $S_{\varepsilon}$ relative to $\pi_{\varepsilon}$.

Suppose that $S$ is a compact invariant set relative to $\pi$ and let $\left(M_{p}\right)_{p \in P}$ be a $\prec$-ordered Morse decomposition of $S$ (relative to $\pi$ ). An index filtration 
for $\left(\pi, S,\left(M_{p}\right)_{p \in P}\right)$ is a family $\mathcal{N}=(N(I))_{I \in \mathcal{A}(\prec)}$ of closed subsets of $Y$ such that

(1) for each $I \in \mathcal{A}(\prec)$, the pair $(N(I), N(\emptyset))$ is an FM-index pair in $M(I)$,

(2) for any $I_{1}, I_{2} \in \mathcal{A}(\prec), N\left(I_{1} \cap I_{2}\right)=N\left(I_{1}\right) \cap N\left(I_{2}\right)$ and $N\left(I_{1} \cup I_{2}\right)=$ $N\left(I_{1}\right) \cup N\left(I_{2}\right)$.

Moreover, $\mathcal{N}$ is called strongly $\pi$-admissible if $N(P)$ is strongly $\pi$-admissible.

Let $\mathcal{N}$ be a strongly $\pi$-admissible index filtration for $\left(\pi, S,\left(M_{p}\right)_{p \in P}\right)$. For $J \in \mathcal{I}(\prec)$ the set $M(J)$ is an isolated invariant set and we write $H(J)=H(\pi, J):=H(\pi, M(J))$. If $(I, J) \in \mathcal{I}_{2}(\prec)$, then $(M(I), M(J))$ is an attractor-repeller pair in $M(I J)$, where $I J:=I \cup J$. Hence there is the corresponding homology index sequence

$$
\longrightarrow H_{q}(I) \stackrel{i_{I, J}}{\longrightarrow} H_{q}(I J) \stackrel{p_{I, J}}{\longrightarrow} H_{q}(J) \stackrel{\partial_{I, J}}{\longrightarrow} H_{q-1}(I) \longrightarrow
$$

of $(\pi, M(I J), M(I), M(J))$. Using the filtration $\mathcal{N}$ one proves that for every triple $(I, J, K) \in \mathcal{I}_{3}(\prec)$ the following diagram, made up of the four homology index sequences defined by $(I, J, K)$, commutes:

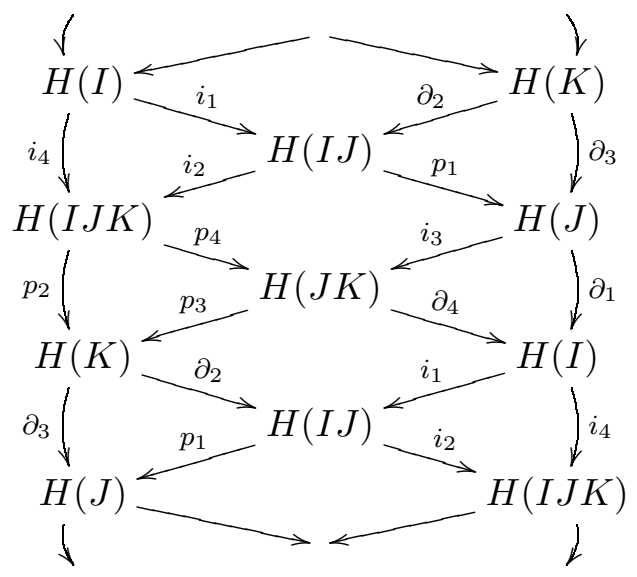

The collection of all the homology indices $H(\pi, M(J)), J \in \mathcal{I}(\prec)$, and all the maps $i_{I, J}, p_{I, J}$ and $\partial_{I, J},(I, J) \in \mathcal{I}_{2}(\prec)$, is called the homology index braid of $\left(\pi, S,\left(M_{p}\right)_{p \in P}\right)$ and is denoted by $\mathcal{H}\left(\pi, S,\left(M_{p}\right)_{p \in P}\right)$.

For the rest of this section assume that, for $i=1,2, \pi_{i}$ is a local semiflow on the metric space $Y_{i}, S_{i}$ is an isolated invariant set and $\left(M_{p, i}\right)_{p \in P}$ is a $\prec-$ ordered Morse decomposition of $S_{i}$ relative to $\pi_{i}$. Write $M_{i}(I)=M_{\pi_{i}, S_{i}}(I)$, $H_{i}(I)=H\left(\pi_{i}, M_{i}(I)\right)$ and $\mathcal{H}_{i}:=\mathcal{H}\left(\pi_{i}, S_{i},\left(M_{p, i}\right)_{p \in P}\right)$, for $i=1,2$ and $I \in$ $\mathcal{I}(\prec)$. 
Suppose $\theta:=(\theta(J))_{J \in \mathcal{I}(\prec)}$ is a family $\theta(J): H_{1}(J) \rightarrow H_{2}(J), J \in \mathcal{I}(\prec)$, of maps such that, for all $(I, J) \in \mathcal{I}_{2}(\prec)$, the diagram

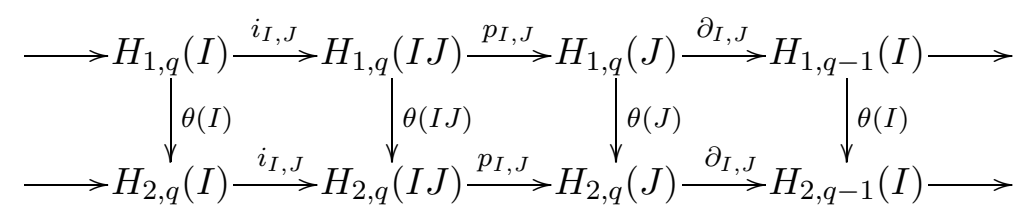

commutes. Then we say that $\theta$ is a morphism from $\mathcal{H}_{1}$ to $\mathcal{H}_{2}$ and we write $\theta: \mathcal{H}_{1} \rightarrow \mathcal{H}_{2}$. If each $\theta(J)$ is an isomorphism, then we say that $\theta$ is an isomorphism and that $\mathcal{H}_{1}$ and $\mathcal{H}_{2}$ are isomorphic homology index braids, and we write $\mathcal{H}_{1} \cong \mathcal{H}_{2}$.

We may analogously define the concept of cohomology index braids and morphisms between them: just use cohomology instead of homology and reverse all the arrows.

Proposition 2.7. Let $X$ and $X^{\prime}$ be metric spaces, $\pi$ be a local semiflow on $X$ and $\pi^{\prime}$ be a local semiflow on $X^{\prime}$. Let $\gamma: X \rightarrow X^{\prime}$ be a homeomorphism which conjugates $\pi$ with $\pi^{\prime}$. Let $S$ be an isolated $\pi$-invariant set which has a strongly $\pi$-admissible isolating neighborhood, and $\left(M_{p}\right)_{p \in P}$ be a Morse decomposition of $S$ relative to $\pi$. Set $S^{\prime}=\gamma(S)$ and $M_{p}^{\prime}=\gamma\left(M_{p}\right), p \in P$.

Then $S^{\prime}$ is an isolated $\pi^{\prime}$-invariant set which has a strongly $\pi^{\prime}$-admissible isolating neighborhood, and $\left(M_{p}^{\prime}\right)_{p \in P}$ is a Morse decomposition of $S^{\prime}$ relative to $\pi^{\prime}$. Moreover,

$$
\mathcal{H}\left(\pi, S,\left(M_{p}\right)_{p \in P}\right) \cong \mathcal{H}\left(\pi^{\prime}, S^{\prime},\left(M_{p}^{\prime}\right)_{p \in P}\right)
$$

Proof. Intuitively, this result is clear. A rigorous proof follows by an application of [8, Theorem 3.2] in the case of homology, and of [18, Theorem 4.2] in the case of cohomology.

We will require the following singular continuation principle for homology index braids and connection matrices established in [7].

TheOREm 2.8 ([7, Theorem 3.10]). Assume the hypotheses (and thus also the conclusions) of Theorem 2.6 hold and let $\widetilde{\varepsilon}>0$ be as in that theorem. Fix $\left.\widetilde{\beta}_{0} \in\right] 0, \beta\left[\right.$. Suppose that there exists a $\beta_{0}>0$ such that for all $\left.\left.\varepsilon \in\right] 0, \varepsilon_{0}\right]$ and all $\left.\eta \in] 0, \beta_{0}\right]$ the set $\mathrm{Cl}_{Y_{\varepsilon}}\left(B_{\varepsilon}\left(\theta_{\varepsilon}, \eta\right)\right)$ is contractible. Then there exists an $\left.\left.\varepsilon_{\mathrm{c}} \in\right] 0, \widetilde{\varepsilon}\right]$ such that

$$
\left.\left.\left.\mathcal{H}\left(\pi_{0}, S_{0},\left(M_{p}\right)_{p \in P}\right) \cong \mathcal{H}\left(\pi_{\varepsilon}, S_{\varepsilon},\left(M_{p, \varepsilon}\right)_{p \in P}\right)\right), \quad \varepsilon \in\right] 0, \varepsilon_{\mathrm{c}}\right] .
$$

Theorem 2.8 refines the corresponding singular Conley index continuation principle established in [2].

REMARK 2.9. The above results were originally proved for singular homology. Analogous definitions and results hold for the Alexander-Spanier cohomology (with coefficients in $G$ ) with the obvious modifications (cf. [6]). 
3. A finite-dimensional perturbation problem. In this section we state and prove a continuation principle for homology index braids and connection matrices for a class of singularly perturbed ordinary differential equations defined on a finite-dimensional space (cf. Theorems 3.1 and 3.6). Throughout this section, we assume the reader's familiarity with the results from $[17$, Section 3].

Let $(E,|\cdot|)$ be a finite-dimensional Banach space. Given a $C^{1}$-map $g: E \rightarrow E$ and $\varepsilon>0$ let $\pi_{\varepsilon, g}$ be the local (semi)flow generated by the following ordinary differential equation on $E \times E$ :

$$
\dot{u}=v, \quad \dot{v}=(1 / \varepsilon)(-v+g(u)), \quad(u, v) \in E .
$$

Furthermore, let $\pi_{g}^{\prime}$ be the local (semi)flow on $E$ generated by the following ordinary differential equation on $E$ :

$$
\dot{u}=g(u), \quad u \in E .
$$

One of the goals of this section is to prove the following result.

THEOREM 3.1. Let $N^{\prime} \subset E$ be a compact isolating neighborhood relative to $\pi_{g}^{\prime}$. Set $S^{\prime}:=\operatorname{Inv}_{\pi_{g}^{\prime}}\left(N^{\prime}\right)$ and let $\left(M_{p}^{\prime}\right)_{p \in P}$ be a $\prec$-ordered Morse decomposition for $S^{\prime}$ relative to $\pi_{g}^{\prime}$. For each $p \in P$, let $V_{p}^{\prime} \subset N^{\prime}$ be closed in $E$ such that $M_{p}^{\prime}=\operatorname{Inv}_{\pi_{g}^{\prime}}\left(V_{p}^{\prime}\right) \subset \operatorname{Int}_{E}\left(V_{p}^{\prime}\right)$. For all $\alpha>0, \varepsilon>0$ and $p \in P$, set $S_{\varepsilon, \alpha}:=\operatorname{Inv}_{\pi_{\varepsilon, g}}\left(N_{\alpha}^{\prime}\right)$ and $M_{p, \varepsilon, \alpha}:=\operatorname{Inv}_{\pi_{\varepsilon, g}}\left(V_{p, \alpha}^{\prime}\right)$, where

$$
\begin{aligned}
N_{\alpha}^{\prime}=N_{\alpha, g}^{\prime} & :=\left\{(u, v) \mid u \in N^{\prime} \text { and }|v-g(u)| \leq \alpha\right\}, \\
V_{p, \alpha}^{\prime}=V_{p, \alpha, g}^{\prime} & :=\left\{(u, v) \mid u \in V_{p}^{\prime} \text { and }|v-g(u)| \leq \alpha\right\} .
\end{aligned}
$$

Then for every $\alpha \in] 0, \infty\left[\right.$, there exists an $\left.\varepsilon_{0}=\varepsilon_{0}(\alpha) \in\right] 0, \infty[$ such that for every $\left.\varepsilon \in] 0, \varepsilon_{0}\right]$, the set $N_{\alpha}^{\prime}$ is an isolating neighborhood of $S_{\varepsilon, \alpha}$ relative to $\pi_{\varepsilon, g}$, and for every $p \in P, V_{p, \alpha}^{\prime}$ is an isolating neighborhood of $M_{p, \varepsilon, \alpha}$ relative to $\pi_{\varepsilon, g}$. Moreover, the family $\left(M_{p, \varepsilon, \alpha}\right)_{p \in P}$ is a $\prec$-ordered Morse decomposition for $S_{\varepsilon, \alpha}$ relative to $\pi_{\varepsilon, g}$ and

$$
\mathcal{H}\left(\pi_{\varepsilon, g}, S_{\varepsilon, \alpha},\left(M_{p, \varepsilon, \alpha}\right)_{p \in P}\right) \cong \mathcal{H}\left(\pi_{g}^{\prime}, S^{\prime},\left(M_{p}^{\prime}\right)_{p \in P}\right) .
$$

The proof of Theorem 3.1 will be based on Theorem 2.8. We proceed similarly to the proof of [17, Theorem 3.1]. Let $U$ be a bounded open neighborhood of $N^{\prime}$ and $\widetilde{g}: E \rightarrow E$ be a $C^{1}$-map such that $g|U=\widetilde{g}| U$ and $\sup _{u \in E}(|\widetilde{g}(u)|+|D \widetilde{g}(u)|)<\infty$. The existence of $\widetilde{g}$ follows since $E$ is finitedimensional.

Since the differential equations defining $\pi_{\varepsilon, g}$ and $\pi_{\varepsilon, \tilde{g}}$ coincide on the open neighborhood $U \times E$ of $N_{\alpha}^{\prime}$ in $E \times E$ it follows that $N_{\alpha}^{\prime}$ (resp. $V_{p, \alpha}^{\prime}$ ) is an isolating neighborhood relative to $\pi_{\varepsilon, g}$ if and only if $N_{\alpha}^{\prime}$ (resp. $V_{p, \alpha}^{\prime}$ ) is an isolating neighborhood relative to $\pi_{\varepsilon, \widetilde{g}}$ and

$$
\begin{aligned}
\operatorname{Inv}_{\pi_{\varepsilon, g}}\left(N_{\alpha}^{\prime}\right) & =S_{\varepsilon, \alpha}=\operatorname{Inv}_{\pi_{\varepsilon, \tilde{g}}}\left(N_{\alpha}^{\prime}\right), \\
\operatorname{Inv}_{\pi_{\varepsilon, g}}\left(V_{p, \alpha}^{\prime}\right) & =M_{p, \varepsilon, \alpha}=\operatorname{Inv}_{\pi_{\varepsilon, \tilde{g}}}\left(V_{p, \alpha}^{\prime}\right), \quad p \in P .
\end{aligned}
$$


Moreover, $\left(M_{p, \varepsilon, \alpha}\right)_{p \in P}$ is a $\prec$-ordered Morse decomposition for $S_{\varepsilon, \alpha}$ relative to $\pi_{\varepsilon, g}$ if and only if $\left(M_{p, \varepsilon, \alpha}\right)_{p \in P}$ is a $\prec$-ordered Morse decomposition for $S_{\varepsilon, \alpha}$ relative to $\pi_{\varepsilon, \tilde{g}}$.

Similarly, $N^{\prime}$ (resp. $V_{p}^{\prime}$ ) is an isolating neighborhood relative to $\pi_{g}^{\prime}$ if and only if $N^{\prime}$ (resp. $V_{p}^{\prime}$ ) is an isolating neighborhood relative to $\pi_{\widetilde{g}}^{\prime}$ and

$$
\operatorname{Inv}_{\pi_{g}^{\prime}}\left(N^{\prime}\right)=S^{\prime}=\operatorname{Inv}_{\pi_{\tilde{g}}^{\prime}}\left(N^{\prime}\right), \quad \operatorname{Inv}_{\pi_{g}^{\prime}}\left(V_{p}^{\prime}\right)=M_{p}^{\prime}=\operatorname{Inv}_{\pi_{\tilde{g}}^{\prime}}\left(V_{p}^{\prime}\right), \quad p \in P .
$$

Furthermore, $\left(M_{p}^{\prime}\right)_{p \in P}$ is a $\prec$-ordered Morse decomposition for $S^{\prime}$ relative to $\pi_{g}^{\prime}$ if and only if $\left(M_{p}^{\prime}\right)_{p \in P}$ is a $\prec$-ordered Morse decomposition for $S^{\prime}$ relative to $\pi_{\tilde{g}}^{\prime}$. Therefore,

$$
\mathcal{H}\left(\pi_{\varepsilon, g}, S_{\varepsilon, \alpha},\left(M_{p, \varepsilon, \alpha}\right)_{p \in P}\right) \cong \mathcal{H}\left(\pi_{g}^{\prime}, S^{\prime},\left(M_{p}^{\prime}\right)_{p \in P}\right)
$$

if and only if

$$
\mathcal{H}\left(\pi_{\varepsilon, \widetilde{g}}, S_{\varepsilon, \alpha},\left(M_{p, \varepsilon, \alpha}\right)_{p \in P}\right) \cong \mathcal{H}\left(\pi_{\widetilde{g}}^{\prime}, S^{\prime},\left(M_{p}^{\prime}\right)_{p \in P}\right) .
$$

Hence we may assume, without loss of generality, that

$$
C:=\sup _{u \in E}(|g(u)|+|D g(u)|)<\infty .
$$

In particular, $g$ is globally Lipschitzian and so both $\pi_{\varepsilon, g}, \varepsilon>0$, and $\pi_{g}^{\prime}$ are global semiflows. We write $\pi_{\varepsilon}:=\pi_{\varepsilon, g}, \varepsilon>0$, and $\pi^{\prime}:=\pi_{g}^{\prime}$ for short.

Notice that the map $\Phi: E \times E \rightarrow E \times E, \Phi(u, v)=(u, w):=(u, v-g(u))$ is a $C^{1}$-diffeomorphism with inverse $\Phi^{-1}$ given by $\Phi^{-1}(u, w)=(u, v):=(u, w+$ $g(u))$. Let $\widetilde{\pi}_{\varepsilon}$ be the conjugate of $\pi_{\varepsilon}$ via $\Phi$, i.e. $(u, w) \widetilde{\pi}_{\varepsilon} t:=\Phi\left(\left(\Phi^{-1}(u, w)\right) \pi_{\varepsilon} t\right)$, $(u, w) \in E \times E, t \in\left[0, \infty\left[\right.\right.$. Note that $\widetilde{\pi}_{\varepsilon}$ is the semiflow generated by the equation

$$
\dot{u}=w+g(u), \quad \dot{w}=-(1 / \varepsilon) w-D g(u)(w+g(u)) .
$$

Let $B_{\alpha}$ be the closed ball in $E$ with radius $\alpha$ centered at zero. It follows that $\Phi\left(N_{\alpha}^{\prime}\right)=N^{\prime} \times B_{\alpha}\left(\operatorname{resp} . \Phi\left(V_{p, \alpha}^{\prime}\right)=V_{p}^{\prime} \times B_{\alpha}, p \in P\right)$.

We have the following

Proposition 3.2. For every $\alpha \in] 0, \infty\left[\right.$, there exists an $\varepsilon_{0}=\varepsilon_{0}(\alpha) \in$ ] $0, \infty[$ such that for all $\left.\varepsilon \in] 0, \varepsilon_{0}\right]$ and $p \in P$, the set $N^{\prime} \times B_{\alpha}\left(\right.$ resp. $\left.V_{p}^{\prime} \times B_{\alpha}\right)$ is an isolating neighborhood of $\widetilde{S}_{\varepsilon, \alpha}\left(\right.$ resp. $\left.\widetilde{M}_{p, \varepsilon, \alpha}\right)$ relative to $\widetilde{\pi}_{\varepsilon}$, where

$$
\widetilde{S}_{\varepsilon, \alpha}=\operatorname{Inv}_{\widetilde{\pi}_{\varepsilon}}\left(N^{\prime} \times B_{\alpha}\right), \quad \widetilde{M}_{p, \varepsilon, \alpha}=\operatorname{Inv}_{\widetilde{\pi}_{\varepsilon}}\left(V_{p}^{\prime} \times B_{\alpha}\right), \quad p \in P .
$$

Moreover, the family $\left(\widetilde{M}_{p, \varepsilon, \alpha}\right)_{p \in P}$ is a $\prec$-ordered Morse decomposition for $\widetilde{S}_{\varepsilon, \alpha}$ relative to $\widetilde{\pi}_{\varepsilon}$ and

$$
\mathcal{H}\left(\widetilde{\pi}_{\varepsilon}, \widetilde{S}_{\varepsilon, \alpha},\left(\widetilde{M}_{p, \varepsilon, \alpha}\right)_{p \in P}\right) \cong \mathcal{H}\left(\pi^{\prime}, S^{\prime},\left(M_{p}^{\prime}\right)_{p \in P}\right) .
$$

Proof. Define $X_{0}=Y_{\varepsilon}=E, \theta_{\varepsilon}=0$ and $d_{0}\left(u, u^{\prime}\right)=d_{\varepsilon}\left(u, u^{\prime}\right)=\left|u-u^{\prime}\right|$ for all $\varepsilon>0$ and $u, u^{\prime} \in E$. It follows that $N^{\prime} \times B_{\alpha}=\left[N^{\prime}\right]_{\varepsilon, \alpha}$ for all $\varepsilon>0$ and $\alpha>0$. Notice that $\mathrm{Cl}_{Y_{\varepsilon}}\left(B_{\varepsilon}\left(\theta_{\varepsilon}, \alpha\right)\right)=B_{\alpha}$ for all $\varepsilon>0$ and 
$\alpha>0$, and so $\mathrm{Cl}_{Y_{\varepsilon}}\left(B_{\varepsilon}\left(\theta_{\varepsilon}, \alpha\right)\right)$ is contractible. The proof of [17, Lemma 3.6], shows that the set $N^{\prime}$ is singularly strongly admissible with respect to $\alpha$ and the family $\left(\widetilde{\pi}_{\varepsilon}\right)_{\varepsilon>0}$ singularly converges to $\pi^{\prime}$. Now an application of Theorems 2.6 and 2.8 completes the proof.

Proposition 3.3. Let $\alpha \in] 0, \infty\left[\right.$ and let $\left.\varepsilon_{0}=\varepsilon_{0}(\alpha) \in\right] 0, \infty[$ be as in Proposition 3.2. Then for every $\left.\varepsilon \in] 0, \varepsilon_{0}\right]$ and $p \in P$, the set $N_{\alpha}^{\prime}$ (resp. $\left.V_{p, \alpha}^{\prime}\right)$ is an isolating neighborhood of $S_{\varepsilon, \alpha}$ (resp. $\left.M_{p, \varepsilon, \alpha}\right)$ relative to $\pi_{\varepsilon}$, the family $\left(M_{p, \varepsilon, \alpha}\right)_{p \in P}$ is a $\prec$-ordered Morse decomposition for $S_{\varepsilon, \alpha}$ relative to $\pi_{\varepsilon}$ and

$$
\mathcal{H}\left(\pi_{\varepsilon}, S_{\varepsilon, \alpha},\left(M_{p, \varepsilon, \alpha}\right)_{p \in P}\right) \cong \mathcal{H}\left(\widetilde{\pi}_{\varepsilon}, \widetilde{S}_{\varepsilon, \alpha},\left(\widetilde{M}_{p, \varepsilon, \alpha}\right)_{p \in P}\right) .
$$

Proof. This follows from Proposition 2.7.

Proof of Theorem 3.1. This follows from Propositions 3.3 and 3.2.

We shall now generalize Theorem 3.1 to comprise isolating neighborhoods which are more general than $N_{\alpha}^{\prime}$. As in [17], define $\mathcal{T}_{g}$ to be the set of all functions $z: \mathbb{R} \rightarrow E \times E$ such that there is a full bounded solution $u: \mathbb{R} \rightarrow E$ of $\pi_{g}^{\prime}$ so that $z(t)=(u(t), g(u(t)))$ for all $t \in \mathbb{R}$. Thus, defining the map $\Gamma_{g}: E \rightarrow E \times E$ by $\Gamma_{g}(\xi)=(\xi, g(\xi)), \xi \in E$, we see that $\mathcal{T}_{g}$ is the set of all functions $z: \mathbb{R} \rightarrow E \times E$ such that there is a full bounded solution $u: \mathbb{R} \rightarrow E$ of $\pi_{g}^{\prime}$ with $z=\Gamma_{g} \circ u$.

Proposition 3.4. Set $\pi_{g}^{\prime}:=\pi^{\prime}$ and $\Gamma:=\Gamma_{g}$. Let $K^{\prime}$ be a compact isolated invariant set relative to $\pi^{\prime}$. Then $\Gamma\left(K^{\prime}\right)$ is a $\mathcal{T}_{g}$-isolated invariant set. Let $N$ be a bounded $\mathcal{T}_{g}$-isolating neighborhood of $\Gamma\left(K^{\prime}\right)$. Let $\left.\beta \in\right] 0, \infty[$ and $B_{\beta}^{\prime}$ be the closed $\beta$-neighborhood of $K^{\prime}$ in $E$. For each $\left.\alpha \in\right] 0, \infty[$, define

$$
B_{\beta, \alpha}^{\prime}:=\left\{(u, v)\left|u \in B_{\beta}^{\prime},\right| v-g(u) \mid \leq \alpha\right\} .
$$

Then there exist an $\alpha \in] 0, \infty[$ and $a \beta \in] 0, \infty\left[\right.$ such that $B_{\beta}^{\prime}$ is an isolating neighborhood of $K^{\prime}$ relative to $\pi^{\prime}$ and $B_{\beta, \alpha}^{\prime} \subset N$. Moreover, there exists an $\left.\varepsilon_{1} \in\right] 0, \infty[$ such that for every $\left.\varepsilon \in] 0, \varepsilon_{1}\right], \operatorname{Inv}_{\pi_{\varepsilon}}\left(B_{\beta, \alpha}^{\prime}\right)=\operatorname{Inv}_{\pi_{\varepsilon}}(N)$.

Proof. This follows from [17, Lemma 3.8 and proof of Theorem 3.9].

We also need the following auxiliary result:

Proposition 3.5. Set $\pi_{g}^{\prime}:=\pi^{\prime}$ and $\Gamma:=\Gamma_{g}$. Let $K^{\prime}$ be a compact isolated invariant set relative to $\pi^{\prime}$ and $K:=\Gamma\left(K^{\prime}\right)$. Let $\mathcal{T}$ be the set of all $(u, v) \in \mathcal{T}_{g}$ such that $(u(t), v(t)) \in K$ for all $t \in \mathbb{R}$. Moreover, let $\left(M_{p}^{\prime}\right)_{p \in P}$ be $a \prec$-ordered Morse decomposition of $K^{\prime}$ relative to $\pi^{\prime}$ and define $M_{p, \Gamma}:=$ $\Gamma\left(M_{p}^{\prime}\right), p \in P$. For every $I \in \mathcal{I}(\prec)$ set

$$
M^{\prime}(I):=\bigcup_{p, q \in I} \operatorname{CS}_{\pi^{\prime}, K^{\prime}}\left(M_{p}^{\prime}, M_{q}^{\prime}\right)
$$


and $M_{\Gamma}(I):=\Gamma\left(M^{\prime}(I)\right)$. Then $\left(M_{p, \Gamma}\right)_{p \in P}$ is a $\prec$-ordered $\mathcal{T}$-Morse decomposition and

$$
M_{\Gamma}(I)=\bigcup_{p, q \in I} \operatorname{CS}_{\mathcal{T}}\left(M_{p}, M_{q}\right), \quad I \in \mathcal{I}(\prec) .
$$

Moreover, the sets $K$ and $M_{p, \Gamma}, p \in P$, and $M_{\Gamma}(I), I \in \mathcal{I}(\prec)$, are $\mathcal{T}_{g}$-isolated invariant sets.

Proof. Write $M_{p}=M_{p, \Gamma}$ and $M(I)=M_{\Gamma}(I)$ for short. We see either directly or using [4, Corollary 3.6] that $M_{p}^{\prime}, p \in P$, and $M^{\prime}(I), I \in \mathcal{I}(\prec)$, are compact isolated invariant sets relative to $\pi^{\prime}$. By [17, Lemma 3.8], the sets $K, M_{p}, p \in P$, and $M(I), I \in \mathcal{I}(\prec)$, are $\mathcal{T}_{g}$-isolated invariant sets. Moreover, the sets $M_{p}$ are closed in $E \times E$ (being compact in $E \times E$ ) and pairwise disjoint ( $\Gamma$ being one-to-one). If $p \in P$ and $(\bar{u}, \bar{v}) \in M_{p}$ are arbitrary, then $\bar{u} \in M_{p}^{\prime}$ so there is a full solution $u$ of $\pi^{\prime}$ lying in $M_{p}^{\prime}$ with $\bar{u}=u(0)$. Thus $u$ lies in $K^{\prime}$ and so $z:=\Gamma \circ u$ lies in $M_{p} \subset K$ and is an element of $\mathcal{T}_{g}$. Hence $z \in \mathcal{T}, z$ lies in $M_{p}$ and $z(0)=(\bar{u}, \bar{v})$. It follows that $M_{p}$ is $\mathcal{T}$-invariant.

Let $(u, v) \in \mathcal{T}$ be arbitrary. Then $u$ is a full solution of $\pi^{\prime}$ lying in $K^{\prime}$. Thus either $u$ lies in $M_{k}^{\prime}$ for some $k \in P$, which implies that $(u, v)$ lies in $M_{k}$, or else there are $k, l \in P$ with $k \prec l, \alpha(u) \subset M_{l}^{\prime}$ and $\omega(u) \subset M_{k}^{\prime}$. In the latter case it is clear from the continuity of $\Gamma$ that $\alpha(u, v) \subset M_{l}$ and $\omega(u, v) \subset M_{k}$. We have proved that $\left(M_{p}\right)_{p \in P}$ is a $\prec$-ordered $\mathcal{T}$-Morse decomposition.

Now let $p, q \in P$ be arbitrary. We claim that

$$
\Gamma\left(\mathrm{CS}_{\pi^{\prime}, K^{\prime}}\left(M_{p}^{\prime}, M_{q}^{\prime}\right)\right)=\mathrm{CS}_{\mathcal{T}}\left(M_{p}, M_{q}\right) .
$$

If $u$ is a full solution of $\pi^{\prime}$ lying in $K^{\prime}$ with $\alpha(u) \subset M_{p}^{\prime}$ and $\omega(u) \subset M_{q}^{\prime}$ then, clearly, $z:=\Gamma \circ u \in \mathcal{T}_{g}, z$ lies in $K, \alpha(z) \subset M_{p}$ and $\omega(z) \subset M_{q}$. Thus

$$
\Gamma\left(\mathrm{CS}_{\pi^{\prime}, K^{\prime}}\left(M_{p}^{\prime}, M_{q}^{\prime}\right)\right) \subset \mathrm{CS}_{\mathcal{T}}\left(M_{p}, M_{q}\right) .
$$

Conversely, if $z \in \mathcal{T}$ with $\alpha(z) \subset M_{p}$ and $\omega(z) \subset M_{q}$ then $z=\Gamma \circ u$, where $u$ is a full solution of $\pi^{\prime}$ lying in $K^{\prime}$. Clearly, $\alpha(u) \subset M_{p}^{\prime}$ and $\omega(u) \subset M_{q}^{\prime}$. Hence

$$
\Gamma\left(\mathrm{CS}_{\pi^{\prime}, K^{\prime}}\left(M_{p}^{\prime}, M_{q}^{\prime}\right)\right) \supset \mathrm{CS}_{\mathcal{T}}\left(M_{p}, M_{q}\right) .
$$

This proves our claim, which, in turn, immediately implies 3.4.

The generalization of Theorem 3.1 reads as follows:

TheOREM 3.6. Assume the hypotheses (and hence the conclusions) of Proposition 3.5. Let $N$ be a bounded $\mathcal{T}_{g}$-isolating neighborhood of $K$ and $N_{p} \subset N$ be a $\mathcal{T}_{g}$-isolating neighborhood of $M_{p, \Gamma}, p \in P$. For $\left.\varepsilon \in\right] 0, \infty[$, set $\pi_{\varepsilon, g}:=\pi_{\varepsilon}, K_{\varepsilon}:=\operatorname{Inv}_{\pi_{\varepsilon}}(N)$, and $M_{p, \varepsilon, \Gamma}:=\operatorname{Inv}_{\pi_{\varepsilon}}\left(N_{p}\right), p \in P$.

Then there is an $\left.\widetilde{\varepsilon}_{0} \in\right] 0, \infty[$ such that for every $\left.\varepsilon \in] 0, \widetilde{\varepsilon}_{0}\right], N$ is an isolating neighborhood of $K_{\varepsilon}$ relative to $\pi_{\varepsilon}$, and for every $p \in P, N_{p}$ is an isolating neighborhood of $M_{p, \varepsilon, \Gamma}$ relative to $\pi_{\varepsilon}$. Moreover, the family $\left(M_{p, \varepsilon, \Gamma}\right)_{p \in P}$ is a 
$\prec$-ordered Morse decomposition of $K_{\varepsilon}$ and

$$
\mathcal{H}\left(\pi_{\varepsilon}, K_{\varepsilon},\left(M_{p, \varepsilon, \Gamma}\right)_{p \in P}\right) \cong \mathcal{H}\left(\pi^{\prime}, K^{\prime},\left(M_{p}^{\prime}\right)_{p \in P}\right) .
$$

Proof. Let $\beta \in] 0, \infty\left[\right.$. Let $B_{\beta}^{\prime}$ be the closed $\beta$-neighborhood of $K^{\prime}$ in $E$. For $p \in P$, let $B_{p, \beta}^{\prime}$ be the closed $\beta$-neighborhood of $M_{p}^{\prime}$ in $E$. For each $\alpha \in] 0, \infty[$, define

$$
\begin{aligned}
B_{\beta, \alpha}^{\prime} & :=\left\{(u, v)\left|u \in B_{\beta}^{\prime},\right| v-g(u) \mid \leq \alpha\right\}, \\
B_{p, \beta, \alpha}^{\prime} & :=\left\{(u, v)\left|u \in B_{p, \beta}^{\prime},\right| v-g(u) \mid \leq \alpha\right\}, \quad p \in P .
\end{aligned}
$$

Proposition 3.4 implies that there exist an $\alpha \in] 0, \infty[$ and a $\beta \in] 0, \infty[$ such that $B_{\beta}^{\prime}$ is an isolating neighborhood of $K^{\prime}$ relative to $\pi^{\prime}$ and $B_{p, \beta}^{\prime}$ is an isolating neighborhood of $M_{p}^{\prime}$ relative to $\pi^{\prime}$ for $p \in P$. Moreover, $B_{\beta, \alpha}^{\prime} \subset N$ and $B_{p, \beta, \alpha}^{\prime} \subset N_{p}$ for $p \in P$. Furthermore, there exists an $\left.\varepsilon_{1} \in\right] 0, \infty[$ such that for every $\left.\varepsilon \in] 0, \varepsilon_{1}\right], \operatorname{Inv}_{\pi_{\varepsilon}}\left(B_{\beta, \alpha}^{\prime}\right)=\operatorname{Inv}_{\pi_{\varepsilon}}(N)$ and $\operatorname{Inv}_{\pi_{\varepsilon}}\left(B_{p, \beta, \alpha}^{\prime}\right)=\operatorname{Inv}_{\pi_{\varepsilon}}\left(N_{p}\right)$, $p \in P$.

Define $N^{\prime}:=B_{\beta}^{\prime}$ and $V_{p}^{\prime}:=B_{p, \beta}^{\prime}, p \in P$. Hence for every $\left.\left.\varepsilon \in\right] 0, \varepsilon_{1}\right]$, we have $N_{\alpha}^{\prime}=B_{\beta, \alpha}^{\prime}$ and $V_{p, \alpha}^{\prime}=B_{p, \beta, \alpha}^{\prime}, p \in P$. Thus

$$
K^{\prime}=\operatorname{Inv}_{\pi^{\prime}}\left(N^{\prime}\right) \subset \operatorname{Int}_{E}\left(N^{\prime}\right), \quad M_{p}^{\prime}=\operatorname{Inv}_{\pi^{\prime}}\left(V_{p}^{\prime}\right) \subset \operatorname{Int}_{E}\left(V_{p}^{\prime}\right), \quad p \in P .
$$

Moreover, for all $\left.\varepsilon \in] 0, \varepsilon_{1}\right]$,

$$
\begin{aligned}
K_{\varepsilon} & =\operatorname{Inv}_{\pi_{\varepsilon}}(N)=\operatorname{Inv}_{\pi_{\varepsilon}}\left(B_{\beta, \alpha}^{\prime}\right)=\operatorname{Inv}_{\pi_{\varepsilon}}\left(N_{\alpha}^{\prime}\right), \\
M_{p, \varepsilon, \Gamma} & =\operatorname{Inv}_{\pi_{\varepsilon}}\left(N_{p}\right)=\operatorname{Inv}_{\pi_{\varepsilon}}\left(B_{p, \beta, \alpha}^{\prime}\right)=\operatorname{Inv}_{\pi_{\varepsilon}}\left(V_{p, \alpha}^{\prime}\right), \quad p \in P .
\end{aligned}
$$

Now an application of Theorem 3.1 together with (3.5) and (3.6) implies that there exists an $\left.\left.\widetilde{\varepsilon}_{0} \in\right] 0, \varepsilon_{1}\right]$ such that for all $\left.\left.\varepsilon \in\right] 0, \widetilde{\varepsilon}_{0}\right]$, the family $\left(M_{p, \varepsilon, \Gamma}\right)_{p \in P}$ is a $\prec$-ordered Morse decomposition of $K_{\varepsilon}$ relative to $\pi_{\varepsilon}$ and

$$
\mathcal{H}\left(\pi_{\varepsilon}, K_{\varepsilon},\left(M_{p, \varepsilon, \Gamma}\right)_{p \in P}\right) \cong \mathcal{H}\left(\pi^{\prime}, K^{\prime},\left(M_{p}^{\prime}\right)_{p \in P}\right)
$$

\section{Continuation of homology index braids for singularly per-} turbed hyperbolic equations. In this section we will state and prove the main result of this paper (cf. Theorem 4.2). After recalling some notations from [17] we state a continuation result for Morse decomposition proved in [17] (cf. Theorem 4.1). Then we state our continuation principle for homology index braids and connection matrices for singularly perturbed hyperbolic equations (cf. Theorem 4.2). We assume the reader's familiarity with the results from [17].

Let $X$ be a real Hilbert space and $A: D(A) \subset X \rightarrow X$ be a positive selfadjoint operator with compact resolvent. Then $A$ is sectorial on $X$ and so it generates the corresponding family $\left(X^{\alpha}\right)_{\alpha \in[0, \infty[}$ of fractional power spaces (cf. [13]). Let $\left(\phi_{\nu}\right)_{\nu \in \mathbb{N}}$ be a complete $X$-orthonormal basis of $X$ consisting of eigenfunctions of $A$. Let $P_{n}: X \rightarrow X$ be the orthogonal projection of 
$X$ onto the subspace spanned by the first $n$ eigenfunctions. Moreover, set $Q_{n}:=I-P_{n}$ where $I$ is the identity map on $X$.

As in [12] and [17], we will assume the following Standing Hypothesis:

$N \in\{1,2,3\}$ and $\Omega \subset \mathbb{R}^{N}$ is a bounded domain with smooth boundary and such that $X=L^{2}(\Omega)$ and $X^{1}$ is continuously included in $H^{2}(\Omega) ; \gamma \in L^{2}(\Omega)$ and $\phi: \mathbb{R} \rightarrow \mathbb{R}$ is a $C^{1}$-function such that, for $N \geq 2$, there are constants $\bar{r}$ and $\bar{C} \in\left[0, \infty\left[\right.\right.$ with $\left|\phi^{\prime}(\xi)\right| \leq \bar{C}\left(1+|\xi|^{\bar{r}}\right)$ for all $\xi \in \mathbb{R}$. If $N=3$ then $\bar{r}<2$.

Define the map $f: X^{1 / 2} \rightarrow X$ by

$$
f(u)=\widehat{\phi}(u)+\gamma, \quad u \in X^{1 / 2} .
$$

We denote by $\pi_{f}^{\prime}$ the local semiflow on $X^{1 / 2}$ generated by the abstract parabolic equation (see [13])

$$
\dot{u}=-A u+f(u), \quad u \in X^{1 / 2} .
$$

For $\varepsilon \in] 0, \infty\left[\right.$ we also consider the local semiflow $\pi_{\varepsilon, f}$ on $X^{1 / 2} \times X$ generated by the damped hyperbolic equation

$$
\dot{u}=v, \quad \dot{v}=(1 / \varepsilon)(-v-A u+f(u)), \quad(u, v) \in X^{1 / 2} \times X .
$$

Let $\Gamma_{f}: X^{1} \rightarrow X^{1 / 2} \times X$ be the map defined by

$$
\Gamma_{f}(u):=(u,-A u+f(u)), \quad u \in X^{1} .
$$

Moreover, we denote by $\mathcal{T}_{f}$ the set of all maps $z: \mathbb{R} \rightarrow X^{1 / 2} \times X$ for which there is a full bounded solution $u$ of $\pi_{f}^{\prime}$ such that $z(t)=\Gamma_{f}(u(t)), t \in \mathbb{R}$. In view of [17, Corollary 4.7], every full bounded solution of $\pi_{f}^{\prime}$ lies in $X^{1}$, so the definition of $\mathcal{T}_{f}$ makes sense.

The following result has been proved in [17].

Theorem 4.1 ([17, Theorem 7.4]). For $\varepsilon \in] 0, \infty\left[\right.$, set $\pi^{\prime}:=\pi_{f}^{\prime}, \pi_{\varepsilon}:=$ $\pi_{\varepsilon, f}$ and $\Gamma:=\Gamma_{f}$. Let $K^{\prime}$ be a compact isolated invariant set relative to $\pi^{\prime}$ and $K:=\Gamma\left(K^{\prime}\right)$. Let $\mathcal{T}$ be the set of all $(u, v) \in \mathcal{T}_{f}$ such that $(u(t), v(t)) \in K$ for all $t \in \mathbb{R}$. Moreover, let $\left(M_{p}^{\prime}\right)_{p \in P}$ a family of subsets of $K^{\prime}$ which is a Morse decomposition of $K^{\prime}$ relative to $\pi^{\prime}$ and let $M_{p}:=\Gamma\left(M_{p}^{\prime}\right), p \in P$. For every $I \in \mathcal{I}(\prec)$ set

$$
M^{\prime}(I):=\bigcup_{p, q \in I} \operatorname{CS}_{\pi^{\prime}, K^{\prime}}\left(M_{p}^{\prime}, M_{q}^{\prime}\right)
$$

and $M(I):=\Gamma\left(M^{\prime}(I)\right)$. Then $\left(M_{p}\right)_{p \in P}$ is a $\prec$-ordered $\mathcal{T}$-Morse decomposition and

$$
M(I)=\bigcup_{p, q \in I} \operatorname{CS}_{\mathcal{T}}\left(M_{p}, M_{q}\right), \quad I \in \mathcal{I}(\prec) .
$$

Moreover, the sets $K, M_{p}, p \in P$ and $M(I), I \in \mathcal{I}(\prec)$, are $\mathcal{T}_{f}$-isolated invariant sets. 
Let $N$ be a bounded $\mathcal{T}_{f}$-isolating neighborhood of $K, V_{p} \subset N$ be a $\mathcal{T}_{f}$-isolating neighborhood of $M_{p}, p \in P$, and $V_{I} \subset N$ be a $\mathcal{T}_{\text {f }}$-isolating neighborhood of $M(I), I \in \mathcal{I}(\prec)$. For $\varepsilon \in] 0, \infty\left[\right.$ set $K_{\varepsilon}:=\operatorname{Inv}_{\pi_{\varepsilon}}(N)$, $M_{p, \varepsilon}:=\operatorname{Inv}_{\pi_{\varepsilon}}\left(V_{p}\right), p \in P$, and $M_{\varepsilon}(I):=\operatorname{Inv}_{\pi_{\varepsilon}}\left(V_{I}\right), I \in \mathcal{I}(\prec)$. Then there is an $\widetilde{\varepsilon} \in] 0, \infty[$ such that for every $\varepsilon \in] 0, \widetilde{\varepsilon}], N$ (resp. $V_{p}$, resp. $\left.V_{I}\right)$ is an isolating neighborhood of $K_{\varepsilon}$ (resp. $M_{p, \varepsilon}$, resp. $M_{\varepsilon}(I)$ ) relative to $\pi_{\varepsilon}$, for all $p \in P$ and all $I \in \mathcal{I}(\prec)$, the family $\left(M_{p, \varepsilon}\right)_{p \in P}$ is a $\prec$-ordered Morse decomposition of $K_{\varepsilon}$ and

$$
M_{\varepsilon}(I)=\bigcup_{p, q \in I} \mathrm{CS}_{\pi_{\varepsilon}, K_{\varepsilon}}\left(M_{p, \varepsilon}, M_{q, \varepsilon}\right), \quad I \in \mathcal{I}(\prec) .
$$

We can now state our main result.

ThEOREM 4.2. Assume the hypotheses (and thus also the conclusions) of Theorem 4.1 hold and let $\widetilde{\varepsilon} \in] 0, \infty[$ be as in that theorem. Then there is an $\left.\left.\varepsilon_{0} \in\right] 0, \widetilde{\varepsilon}\right]$ such that for every $\left.\left.\varepsilon \in\right] 0, \varepsilon_{0}\right]$,

$$
\mathcal{H}\left(\pi_{\varepsilon}, K_{\varepsilon},\left(M_{p, \varepsilon}\right)_{p \in P}\right) \cong \mathcal{H}\left(\pi^{\prime}, K^{\prime},\left(M_{p}^{\prime}\right)_{p \in P}\right) .
$$

Theorem 4.2 refines the corresponding Conley index continuation principle established in [17].

The rest of this section is devoted to the proof of our main theorem. We give the proof for the singular homology case. The Alexander-Spanier cohomology case is analogous.

Theorem 4.2 follows from a series of lemmas. For the rest of this section, for $n \in \mathbb{N}$ and $\theta \in[0,1]$, let $f_{n, \theta}: X^{1 / 2} \rightarrow X$ be defined by

$$
\begin{aligned}
f_{n, \theta} & =(1-\theta) f(u)+\theta P_{n} f\left(P_{n} u\right) \\
& =(1-\theta)(\widehat{\phi}(u)+\gamma)+\theta P_{n}\left(\widehat{\phi}\left(P_{n} u\right)+\gamma\right), \quad u \in X^{1 / 2} .
\end{aligned}
$$

The following lemma has been proved in [17].

Lemma 4.3 ([17, Theorem 4.3 and Proposition 4.4]). Let $N^{\prime} \subset X^{1 / 2}$ be bounded and closed. Furthermore, let $\left(n_{m}\right)_{m}$ be a sequence in $\mathbb{N}$ with $n_{m} \rightarrow \infty$ and $\left(\theta_{m}\right)_{m}$ be an arbitrary sequence in $[0,1]$. For every $m \in \mathbb{N}$, let $u_{m}$ be a full solution of $\pi_{f_{n_{m}, \theta_{m}}}$ lying in $N^{\prime}$. Then there is a sequence $\left(m_{k}\right)_{k}$ with $m_{k} \rightarrow \infty$ and there is a full solution $u$ of $\pi^{\prime}$ lying in $N^{\prime}$ such that $u_{m_{k}}(t) \rightarrow u(t)$ in $X^{1 / 2}$, uniformly for $t$ lying in compact subsets of $\mathbb{R}$.

Lemma 4.4. Let $N^{\prime}$ be bounded and closed in $X^{1 / 2}$ with $K^{\prime}:=\operatorname{Inv}_{\pi^{\prime}}\left(N^{\prime}\right)$ $\subset \operatorname{Int}_{X^{1 / 2}}\left(N^{\prime}\right)$. For each $p \in P$, let $V_{p}^{\prime} \subset N^{\prime}$ be closed in $X^{1 / 2}$ such that $M_{p}^{\prime}=\operatorname{Inv}_{\pi^{\prime}}\left(V_{p}^{\prime}\right) \subset \operatorname{Int}_{X^{1 / 2}}\left(V_{p}^{\prime}\right)$. For $n \in \mathbb{N}, \theta \in[0,1]$ and $p \in P$ define $K_{n, \theta}^{\prime}=\operatorname{Inv}_{\pi_{f_{n, \theta}}^{\prime}}\left(N^{\prime}\right)$ and $M_{p, n, \theta}^{\prime}=\operatorname{Inv}_{\pi_{f_{n, \theta}}^{\prime}}\left(V_{p}^{\prime}\right)$. Then there is an $n_{0} \in \mathbb{N}$ so that whenever $n \geq n_{0}$ and $\theta \in[0,1]$, then $K_{n, \theta}^{\prime} \subset \operatorname{Int}_{X^{1 / 2}}\left(N^{\prime}\right), M_{p, n, \theta}^{\prime} \subset$ 
$\operatorname{Int}_{X^{1 / 2}}\left(V_{p}^{\prime}\right), p \in P$, and the family $\left(M_{p, n, \theta}^{\prime}\right)_{p \in P}$ is a $\prec$-ordered Morse decomposition of $K_{n, \theta}^{\prime}$ relative to $\pi_{f_{n, \theta}}^{\prime}$. Moreover, for all $n \geq n_{0}$ and all $\theta \in[0,1]$,

$$
\mathcal{H}\left(\pi_{f_{n, \theta}}^{\prime}, K_{n, \theta}^{\prime},\left(M_{p, n, \theta}^{\prime}\right)_{p \in P}\right) \cong \mathcal{H}\left(\pi^{\prime}, K^{\prime},\left(M_{p}^{\prime}\right)_{p \in P}\right) .
$$

Proof. This follows from [5, Corollaries 3.9 and 3.10].

Given $n \in \mathbb{N}$ we consider the local semiflow $\pi_{n}^{\prime}$ generated on the finitedimensional space $Y_{n}:=P_{n}\left(X^{1 / 2}\right)=P_{n}(X)$ by the ordinary differential equation

$$
\dot{u}=-A u+P_{n} f\left(P_{n} u\right), \quad u \in Y_{n} .
$$

The local semiflow $\pi_{n}^{\prime}$ is the $n$-Galerkin approximation of $\pi^{\prime}$.

Moreover, let $\pi_{n}^{\prime \prime}$ be the semiflow generated on $Z_{n}:=Q_{n}\left(X^{1 / 2}\right)$ by the evolution equation

$$
\dot{u}=-A u, \quad u \in Z_{n} .
$$

By [17, Proposition 4.2], and its proof, the space $Y_{n}$ is positively invariant relative to the local semiflow $\pi_{f_{n, 1}}^{\prime}$ and every bounded $\pi_{f_{n, 1}}^{\prime}$-invariant set is included in $Y_{n}$ and is $\pi_{n}^{\prime}$-invariant. Moreover, every $\pi_{n}^{\prime}$-invariant set is $\pi_{f_{n, 1}}^{\prime}$-invariant. Setting $K_{n}^{\prime}:=K_{n, 1}^{\prime}$ and $M_{p, n}^{\prime}:=M_{p, n, 1}^{\prime}, p \in P$, we thus see that, whenever $n \geq n_{0}$, where $n_{0} \in \mathbb{N}$ is as in Lemma 4.4, then $K_{n}^{\prime}$ is a compact $\pi_{n}^{\prime}$-invariant set and the family $\left(M_{p, n}^{\prime}\right)_{p \in P}$ is a $\prec$-ordered Morse decomposition of $K_{n}^{\prime}$ relative to $\pi_{n}^{\prime}$. Moreover, the following continuation result has been proved in [5].

Lemma 4.5. Let $n_{0} \in \mathbb{N}$ be as in Lemma 4.4. Then for all $n \geq n_{0}$,

$$
\mathcal{H}\left(\pi_{f_{n, 1}}^{\prime}, K_{n}^{\prime},\left(M_{p, n}^{\prime}\right)_{p \in P}\right) \cong \mathcal{H}\left(\pi_{n}^{\prime}, K_{n}^{\prime},\left(M_{p, n}^{\prime}\right)_{p \in P}\right) .
$$

Now, Lemmas 4.4 and 4.5 imply the following result.

Proposition 4.6 ([5, Theorem 3.11]). Let $n_{0} \in \mathbb{N}$ be as in Lemma 4.4. Then for all $n \geq n_{0}$,

$$
\mathcal{H}\left(\pi^{\prime}, K^{\prime},\left(M_{p}^{\prime}\right)_{p \in P}\right) \cong \mathcal{H}\left(\pi_{n}^{\prime}, K_{n}^{\prime},\left(M_{p, n}^{\prime}\right)_{p \in P}\right) .
$$

Lemma 4.7. Let $\left(\varepsilon_{m}\right)_{m},\left(n_{m}\right)_{m}$ and $\left(\theta_{m}\right)_{m}$ be sequences in $] 0, \infty[, \mathbb{N}$ and $[0,1]$, respectively. Suppose that $\varepsilon_{m} \rightarrow 0$ and $n_{m} \rightarrow \infty$. For each $m \in \mathbb{N}$, let $\left(u_{m}, v_{m}\right)$ be a full solution of $\pi_{\varepsilon_{m}, f_{n_{m}, \theta_{m}}}$ lying in $N$. Then there is a subsequence of $\left(\left(u_{m}, v_{m}\right)\right)_{m}$, denoted again by $\left(\left(u_{m}, v_{m}\right)\right)_{m}$, and there is a full bounded solution $u$ of $\pi^{\prime}$ such that $\left(u_{m}, v_{m}\right) \rightarrow(u, v)$ in $X^{1 / 2} \times X$, uniformly on compact subsets of $\mathbb{R}$. Here, $(u, v)=\Gamma \circ u$.

Proof. This follows from [17, Theorem 5.13].

Lemma 4.8. For $\varepsilon \in] 0, \infty[, n \in \mathbb{N}, \theta \in[0,1], p \in P$ and $I \in \mathcal{I}(\prec)$, define $K_{\varepsilon, n, \theta}=\operatorname{Inv}_{\pi_{\varepsilon, f_{n, \theta}}}(N), M_{p, \varepsilon, n, \theta}=\operatorname{Inv}_{\pi_{\varepsilon, f_{n, \theta}}}\left(V_{p}\right)$ and $M_{\varepsilon, n, \theta}(I)=$ $\operatorname{Inv}_{\pi_{\varepsilon, f_{n, \theta}}}\left(V_{I}\right)$. Let $\left.\widetilde{\varepsilon} \in\right] 0, \infty\left[\right.$ be as in Theorem 4.1. Then there are $n^{\prime} \in \mathbb{N}$ 
and $\left.\left.\varepsilon^{\prime} \in\right] 0, \widetilde{\varepsilon}\right]$ so that whenever $\left.\left.n \geq n^{\prime}, \varepsilon \in\right] 0, \varepsilon^{\prime}\right]$ and $\theta \in[0,1]$, then $K_{\varepsilon, n, \theta} \subset \operatorname{Int}_{X^{1 / 2} \times X}(N), M_{\varepsilon, p, n, \theta} \subset \operatorname{Int}_{X^{1 / 2} \times X}\left(V_{p}\right), p \in P$, and the family $\left(M_{p, \varepsilon, n, \theta}\right)_{p \in P}$ is a

Proof. It is well-known (cf. [3, Theorems 5.3 and 5.5]) that $N$ is strongly

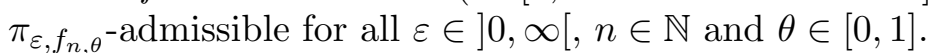

It follows from [17, Lemma 6.4] that there exist an $n_{0}^{\prime} \in \mathbb{N}$ and an $\varepsilon_{0}^{\prime}>0$ such that for all $\left.\left.n \geq n_{0}^{\prime}, \varepsilon \in\right] 0, \varepsilon_{0}^{\prime}\right]$ and $\theta \in[0,1]$ the sets $N, V_{p}, p \in P$, and $V_{I}, I \in \mathcal{I}(\prec)$, are isolating neighborhoods relative to $\pi_{\varepsilon, f_{n, \theta}}$.

Notice that

$$
W \subset N \text { implies that } \operatorname{Inv}_{\mathcal{T}_{f}}(W)=\operatorname{Inv}_{\mathcal{T}}(W) .
$$

Suppose the conclusion of the lemma does not hold. Then for all $n^{\prime} \in \mathbb{N}$ and all $\left.\left.\varepsilon^{\prime} \in\right] 0, \widetilde{\varepsilon}\right]$ there exist an $n \geq n^{\prime}$, an $\left.\left.\varepsilon \in\right] 0, \varepsilon^{\prime}\right]$ and a $\theta \in[0,1]$ such that the family $\left(M_{p, \varepsilon, n, \theta}\right)_{p \in P}$ is not a $\prec$-ordered Morse decomposition of $K_{\varepsilon, n, \theta}$ relative to $\pi_{\varepsilon, f_{n, \theta}}$. Hence there are sequences $\left(\varepsilon_{m}\right)_{m}$ in $] 0, \infty\left[,\left(n_{m}\right)_{m}\right.$ in $\mathbb{N}$ and $\left(\theta_{m}\right)_{m}$ in $[0,1]$ such that $\varepsilon_{m} \rightarrow 0, n_{m} \rightarrow \infty$ as $m \rightarrow \infty$ and, for all $m \in \mathbb{N}$, the family $\left(M_{p, \varepsilon_{m}, n_{m}, \theta_{m}}\right)_{p \in P}$ is not a $\prec$-ordered Morse decomposition of $K_{\varepsilon_{m}, n_{m}, \theta_{m}}$ relative to $\pi_{\varepsilon_{m}, f_{n_{m}, \theta_{m}}}$.

For each $m \in \mathbb{N}$, let $\mathcal{T}_{\varepsilon_{m}, n_{m}, \theta_{m}}$ denote the set of all full solutions of $\pi_{\varepsilon_{m}, f_{n_{m}, \theta_{m}}}$ lying in $N$. It follows that

$$
\begin{aligned}
M_{p, \varepsilon_{m}, n_{m}, \theta_{m}} & =\operatorname{Inv}_{\mathcal{T}_{\mathcal{\varepsilon}_{m}, n_{m}, \theta_{m}}}\left(V_{p}\right), & & p \in P, m \in \mathbb{N}, \\
M_{\varepsilon_{m}, n_{m}, \theta_{m}}(I) & =\operatorname{Inv}_{\mathcal{T}_{\mathcal{\varepsilon}_{m}, n_{m}, \theta_{m}}}\left(V_{I}\right), & & I \in I(\prec), m \in \mathbb{N} .
\end{aligned}
$$

We claim that

$$
\mathcal{T}_{\varepsilon_{m}, n_{m}, \theta_{m}} \rightarrow \mathcal{T} \quad \text { as } m \rightarrow \infty .
$$

In fact, let $\left(m_{k}\right)_{k}$ be an arbitrary sequence in $\mathbb{N}$ with $m_{k} \rightarrow \infty$ and for every $k \in \mathbb{N}$ let $\left(u_{k}, v_{k}\right)$ be a full solution of $\pi_{\varepsilon_{m_{k}}, f_{n_{m_{k}}, \theta_{m_{k}}}}$ lying in $N$. Thus

$$
\sup _{k \in \mathbb{N}} \sup _{t \in \mathbb{R}}\left\|\left(u_{k}(t), v_{k}(t)\right)\right\|_{X^{1 / 2} \times X}<\infty
$$

so, by Lemma 4.7 , there is a subsequence of $\left(\left(u_{k}, v_{k}\right)\right)_{k}$, denoted again by $\left(\left(u_{k}, v_{k}\right)\right)_{k}$, and there is a full bounded solution $u$ of $\pi^{\prime}$ such that $\left(u_{k}, v_{k}\right) \rightarrow$ $(u, v)$ in $X^{1 / 2} \times X$, uniformly on compact subsets of $\mathbb{R}$. Here, $(u, v)=\Gamma \circ u$. Since $N$ is closed in $X^{1 / 2} \times X$, we see that $(u, v)$ lies in $N$, so that $(u, v)$ actually lies in $K$. Thus $(u, v) \in \mathcal{T}$ and claim (4.5) is proved.

We also claim that

$\mathcal{T}$ and $\mathcal{T}_{\varepsilon_{m}, n_{m}, \theta_{m}}, m \in \mathbb{N}$, are compact in $C\left(\mathbb{R} \rightarrow X^{1 / 2} \times X\right)$, translation and cut-and-glue invariant.

Recall that

$$
\mathcal{T}_{\varepsilon_{m}, n_{m}, \theta_{m}}=\mathcal{T}_{\pi_{\varepsilon_{m}, f_{n_{m}, \theta_{m}}}, N} \quad \text { for all } m \in \mathbb{N}
$$


Now (4.7) and [4, Proposition 2.7] imply the part of claim (4.6) concerning $\mathcal{T}_{\varepsilon_{m}, n_{m}, \theta_{m}}, m \in \mathbb{N}$.

In order to prove the compactness of $\mathcal{T}$, let $\left(\left(u_{\kappa}, v_{\kappa}\right)\right)_{\kappa}$ be an arbitrary sequence in $\mathcal{T}$. Thus, for every $\kappa \in \mathbb{N}, u_{\kappa}$ is a full solution of $\pi^{\prime}$ lying in $K^{\prime}$. Now an application of [17, Theorem 4.6] (with $f_{\kappa} \equiv f$ ) shows that there is a sequence $\left(\kappa_{n}\right)_{n}$ in $\mathbb{N}$ with $\kappa_{n} \rightarrow \infty$ and there is a full solution $u$ of $\pi^{\prime}$ lying in $K^{\prime}$ such that $u_{\kappa_{n}} \rightarrow u$ in $X^{1}$, uniformly on compact subsets of $\mathbb{R}$. Thus $\left(u_{\kappa_{n}}, v_{\kappa_{n}}\right) \rightarrow(u, v)$ in $X^{1 / 2} \times X$, uniformly on compact subsets of $\mathbb{R}$, where $(u, v)=\Gamma \circ u$. It follows that $(u, v) \in \mathcal{T}$ and so $\mathcal{T}$ is compact in $C\left(\mathbb{R} \rightarrow X^{1 / 2} \times X\right)$, as claimed. The translation and cut-and-glue invariance of $\mathcal{T}$ is obvious. This completes the proof of (4.6).

An application of [4, Theorem 3.3] together with (4.4)-(4.6) shows that there is an $m_{0} \in \mathbb{N}$ such that for every $m \geq m_{0}$, the family $\left(M_{p, \varepsilon_{m}, n_{m}, \theta_{m}}\right)_{p \in P}$ is a $\prec$-ordered $\mathcal{T}_{\varepsilon_{m}, n_{m}, \theta_{m}}$-Morse decomposition. This is a contradiction. The lemma is proved.

Lemma 4.9. Let $n^{\prime} \in \mathbb{N}$ and $\left.\varepsilon^{\prime} \in\right] 0, \infty[$ be as in Lemma 4.8. Then for all $\left.\left.n \geq n^{\prime}, \varepsilon \in\right] 0, \varepsilon^{\prime}\right]$ and $\theta \in[0,1]$,

$$
\mathcal{H}\left(\pi_{\varepsilon, f_{n, \theta}}, K_{\varepsilon, n, \theta},\left(M_{p, \varepsilon, n, \theta}\right)_{p \in P}\right) \cong \mathcal{H}\left(\pi_{\varepsilon}, K_{\varepsilon},\left(M_{p, \varepsilon}\right)_{p \in P}\right) .
$$

Proof. Let $n \geq n^{\prime}$ and $\left.\left.\varepsilon \in\right] 0, \varepsilon^{\prime}\right]$ be fixed. Let $\left(\theta_{k}\right)_{k}$ be a sequence in $[0,1]$ such that $\theta_{k} \rightarrow \theta_{0}$ in $[0,1]$. Then $f_{n, \theta_{k}}(u) \rightarrow f_{n, \theta_{0}}(u)$ in $X$, locally uniformly in $u \in X^{1 / 2}$. This implies, by the results in [16], that $\pi_{\varepsilon, f_{n, \theta_{k}}} \rightarrow$ $\pi_{\varepsilon, f_{n, \theta_{0}}}$. Moreover, the results in [3] imply that $N$ is $\left(\pi_{f_{n, \theta_{k}}}\right)_{k}$-admissible. Now Lemma 4.8 and the abstract regular continuation principle [5, Theorem 3.7] complete the proof.

Given $\varepsilon \in] 0, \infty\left[\right.$ and $n \in \mathbb{N}$ we consider the local semiflow $\pi_{\varepsilon, n}$ generated on the finite-dimensional space $X_{n}:=P_{n}\left(X^{1 / 2}\right) \times P_{n}(X)$ by the ordinary differential equation

$$
\dot{u}=v, \quad \dot{v}=(1 / \varepsilon)\left(-v-A u+P_{n} f\left(P_{n} u\right)\right), \quad(u, v) \in X_{n} .
$$

The local semiflow $\pi_{\varepsilon, n}$ is the $n$-Galerkin approximation of $\pi_{\varepsilon}$.

Moreover, let $\pi_{\varepsilon, n}^{\prime \prime}$ be the semiflow generated on $W_{n}:=Q_{n}\left(X^{1 / 2}\right) \times$ $Q_{n}(X)$ by the evolution equation

$$
\dot{u}=v, \quad \dot{v}=(1 / \varepsilon)(-v-A u), \quad(u, v) \in W_{n} .
$$

By [17, Proposition 5.3 and its proof], the space $X_{n}$ is positively invariant relative to the local semiflow $\pi_{\varepsilon, f_{n, 1}}$, and every bounded $\pi_{\varepsilon, f_{n, 1}}$-invariant set is included in $X_{n}$ and is $\pi_{\varepsilon, n}$-invariant. Moreover, every $\pi_{\varepsilon, n}$-invariant set is $\pi_{\varepsilon, f_{n, 1}}$-invariant. Setting $K_{\varepsilon, n}:=K_{\varepsilon, n, 1}$ and $M_{p, \varepsilon, n}:=M_{p, \varepsilon, n, 1}, p \in P$, we thus see that, whenever $\left.n \geq n^{\prime}, \varepsilon \in\right] 0, \varepsilon^{\prime}$, where $n^{\prime} \in \mathbb{N}$ and $\left.\varepsilon^{\prime} \in\right] 0, \infty[$ are as in Lemma 4.8 , then $K_{\varepsilon, n}$ is a compact $\pi_{\varepsilon, n}$-invariant set and $\left(M_{p, \varepsilon, n}\right)_{p \in P}$ is a $\prec$-ordered Morse decomposition of $K_{\varepsilon, n}$ relative to $\pi_{\varepsilon, n}$. Furthermore: 
Lemma 4.10. Let $n^{\prime} \in \mathbb{N}$ and $\left.\varepsilon^{\prime} \in\right] 0, \infty[$ be as in Lemma 4.8. Then for $n \geq n^{\prime}$ and $\left.\left.\varepsilon \in\right] 0, \varepsilon^{\prime}\right]$,

$$
\mathcal{H}\left(\pi_{\varepsilon, n}, K_{\varepsilon, n},\left(M_{p, \varepsilon, n}\right)_{p \in P}\right) \cong \mathcal{H}\left(\pi_{\varepsilon, f_{n, 1}}, K_{\varepsilon, n},\left(M_{p, \varepsilon, n}\right)_{p \in P}\right) .
$$

Proof. Fix $\left.\varepsilon \in] 0, \varepsilon^{\prime}\right]$ and $n \geq n^{\prime}$. Let $\mathcal{N}_{\varepsilon, n}^{\prime}=\left(N_{\varepsilon, n}^{\prime}(I)\right)_{I \in \mathcal{A}(\prec)}$ be a strongly $\pi_{\varepsilon, n}$-admissible index filtration for $\left(\pi_{\varepsilon, n}, K_{\varepsilon, n},\left(M_{p, \varepsilon, n}\right)_{p \in P}\right)$. (Strong $\pi_{\varepsilon, n}$-admissibility means, in this finite-dimensional case, simply that $N_{\varepsilon, n}^{\prime}(P)$ is bounded in $X_{n}$.) Let $B=B_{n}$ be the unit ball in $W_{n}$. Since $\left|u \pi_{\varepsilon, n}^{\prime \prime} t\right|_{W_{n}} \leq$ $e^{-\beta_{n} t}|u|_{W_{n}}$ for some $\left.\beta_{n} \in\right] 0, \infty\left[\right.$ and all $u \in W_{n}$ and $t \in[0, \infty[$ it follows that, relative to $\pi_{\varepsilon, n}^{\prime \prime}, B$ is an isolating block for $\{0\}$ with empty exit set, so in particular, $B$ is positively invariant.

We define $N_{\varepsilon, n}(I):=N_{\varepsilon, n}^{\prime}(I)+B \cong N_{\varepsilon, n}^{\prime}(I) \times B, I \in \mathcal{A}(\prec)$. It is now a simple exercise to show that $\mathcal{N}_{\varepsilon, n}=\left(N_{\varepsilon, n}(I)\right)_{I \in \mathcal{A}(\prec)}$ is a strongly $\pi_{\varepsilon, f_{n, 1}}{ }^{-}$ admissible index filtration for $\left(\pi_{\varepsilon, f_{n, 1}}, K_{\varepsilon, n},\left(M_{p, \varepsilon, n}\right)_{p \in P}\right)$. We claim that

$$
\mathcal{H}\left(\pi_{\varepsilon, n}, K_{\varepsilon, n},\left(M_{p, \varepsilon, n}\right)_{p \in P}\right) \cong \mathcal{H}\left(\pi_{\varepsilon, f_{n, 1}}, K_{\varepsilon, n},\left(M_{p, \varepsilon, n}\right)_{p \in P}\right) .
$$

In fact, let $J \in \mathcal{I}(\prec)$ be arbitrary. Choose $I, K \in \mathcal{A}(\prec)$ with $(I, J) \in \mathcal{I}_{2}(\prec)$ and $K=I J$. Let $\phi: N_{\varepsilon, n}^{\prime}(K) / N_{\varepsilon, n}^{\prime}(I) \rightarrow N_{\varepsilon, n}(K) / N_{\varepsilon, n}(I)$ be inclusion induced and $\psi: N_{\varepsilon, n}(K) / N_{\varepsilon, n}(I) \rightarrow N_{\varepsilon, n}^{\prime}(K) / N_{\varepsilon, n}^{\prime}(I)$ be induced by the canonical projection $y+z \mapsto y$ of $X^{1 / 2} \times X=X_{n} \oplus W_{n}$ onto $X_{n}$. It follows that $\psi \circ \phi$ is the identity on $N_{\varepsilon, n}^{\prime}(K) / N_{\varepsilon, n}^{\prime}(I)$ while $\phi \circ \psi$ is homotopic to the identity on $N_{\varepsilon, n}(K) / N_{\varepsilon, n}(I)$ via the homotopy $N_{\varepsilon, n}(K) / N_{\varepsilon, n}(I) \times$ $[0,1] \rightarrow N_{\varepsilon, n}(K) / N_{\varepsilon, n}(I)$ induced by the homotopy $(y+z, \tau) \mapsto y+\tau z$ on $X^{1 / 2} \times X=X_{n} \oplus W_{n}$. The homotopy axiom for singular homology now implies that the map

$$
\theta_{\mathcal{N}_{\varepsilon, n}^{\prime}, \mathcal{N}_{\varepsilon, n}}(J): H\left(\pi_{\varepsilon, n}, M_{\varepsilon, n}(J)\right) \rightarrow H\left(\pi_{\varepsilon, f_{n, 1}}, M_{\varepsilon, n}(J)\right)
$$

induced by $\phi$ is an isomorphism. Claim (4.9) is proved, which in turn concludes the proof of the lemma.

Now, Lemmas 4.9 and 4.10 imply the following proposition.

Proposition 4.11. Let $n^{\prime} \in \mathbb{N}$ and $\left.\varepsilon^{\prime} \in\right] 0, \infty[$ be as in Lemma 4.8 . Then for $n \geq n^{\prime}$ and $\left.\varepsilon \in\right] 0, \varepsilon^{\prime}$,

$$
\mathcal{H}\left(\pi_{\varepsilon, n}, K_{\varepsilon, n},\left(M_{p, \varepsilon, n}\right)_{p \in P}\right) \cong \mathcal{H}\left(\pi_{\varepsilon}, K_{\varepsilon},\left(M_{p, \varepsilon}\right)_{p \in P}\right) .
$$

Lemma 4.12. There exists an $n_{1} \in \mathbb{N}$ such that for every $n \geq n_{1}$, the sets $N$ and $V_{p}, p \in P$, are $\mathcal{T}_{f_{n, 1}}$-isolating neighborhoods of $K_{n}:=\bar{\Gamma}_{f_{n, 1}}\left(K_{n}^{\prime}\right)$ and $M_{p, n}:=\Gamma_{f_{n, 1}}\left(M_{p, n}^{\prime}\right), p \in P$, respectively, where $N^{\prime}$ and $V_{p}^{\prime}, p \in P$, are as in Theorem 4.1 .

Proof. This follows from [17, Theorem 4.11].

We can now give the proof of Theorem 4.2. 
Proof of Theorem 4.2. Let $n_{0} \in \mathbb{N}$ be as in Lemma 4.4, $n^{\prime} \in \mathbb{N}$ be as in Lemma 4.8 and $n_{1} \in \mathbb{N}$ be as in Lemma 4.12. Define $\widetilde{n}:=\max \left\{n_{0}, n^{\prime}, n_{1}\right\}$. Fix $n \geq \widetilde{n}$ arbitrarily. Proposition 4.6 implies that

$$
\mathcal{H}\left(\pi_{n}^{\prime}, K_{n}^{\prime},\left(M_{p, n}^{\prime}\right)_{p \in P}\right) \cong \mathcal{H}\left(\pi^{\prime}, K^{\prime},\left(M_{p}^{\prime}\right)_{p \in P}\right) .
$$

Notice that

$$
\begin{aligned}
K_{n}^{\prime} & =\operatorname{Inv}_{\pi_{f_{n, 1}}^{\prime}}\left(N^{\prime}\right)=\operatorname{Inv}_{\pi_{n}^{\prime}}\left(N^{\prime} \cap Y_{n}\right) \subset \operatorname{Int}_{Y_{n}}\left(N^{\prime} \cap Y_{n}\right), \\
M_{p, n}^{\prime} & =\operatorname{Inv}_{\pi_{f_{n, 1}}^{\prime}}\left(V_{p}^{\prime}\right)=\operatorname{Inv}_{\pi_{n}^{\prime}}\left(V_{p}^{\prime} \cap Y_{n}\right) \subset \operatorname{Int}_{Y_{n}}\left(V_{p}^{\prime} \cap Y_{n}\right), \quad p \in P .
\end{aligned}
$$

Moreover, with the notation introduced in Section 3, it follows from Lemma 4.12 that

$$
\begin{aligned}
& \operatorname{Inv}_{\mathcal{T}_{g_{n}}}\left(N \cap X_{n}\right)=\operatorname{Inv}_{\mathcal{T}_{f_{n, 1}}}(N)=K_{n} \subset \operatorname{Int}_{X^{1 / 2} \times X}(N), \\
& \operatorname{Inv}_{\mathcal{T}_{g_{n}}}\left(V_{p} \cap X_{n}\right)=\operatorname{Inv}_{\mathcal{T}_{f_{n, 1}}}\left(V_{p}\right)=M_{p, n} \subset \operatorname{Int}_{X^{1 / 2} \times X}\left(V_{p}\right), \quad p \in P,
\end{aligned}
$$

where $g_{n}: Y_{n} \rightarrow Y_{n}$ is defined by

$$
g_{n}(u)=-A u+P_{n}(\widehat{\phi}(u)+\gamma), \quad u \in Y_{n} .
$$

Hence

$$
\begin{aligned}
& \operatorname{Inv}_{\mathcal{T}_{g_{n}}}\left(N \cap X_{n}\right) \subset \operatorname{Int}_{X_{n}}\left(N \cap X_{n}\right), \\
& \operatorname{Inv}_{\mathcal{T}_{g_{n}}}\left(V_{p} \cap X_{n}\right) \subset \operatorname{Int}_{X_{n}}\left(V_{p} \cap X_{n}\right), \quad p \in P .
\end{aligned}
$$

It follows from Theorem 3.6 that there exists an $\left.\widetilde{\varepsilon}_{0} \in\right] 0, \infty[$ such that for all $\left.\varepsilon \in] 0, \widetilde{\varepsilon}_{0}\right]$, the family $\left(M_{p, \varepsilon, \Gamma_{g_{n}}}\right)_{p \in P}$ is a $\prec$-ordered Morse decomposition of $\widetilde{K}_{\varepsilon, n}$ and $\mathcal{H}\left(\pi_{\varepsilon, n}, \widetilde{K}_{\varepsilon, n},\left(M_{p, \varepsilon, \Gamma_{n}}\right)_{p \in P}\right) \cong \mathcal{H}\left(\pi_{n}^{\prime}, K_{n}^{\prime},\left(M_{p, n}^{\prime}\right)_{p \in P}\right)$, where

$$
\begin{aligned}
\widetilde{K}_{\varepsilon, n} & =\operatorname{Inv}_{\pi_{\varepsilon, n}}\left(N \cap X_{n}\right)=\operatorname{Inv}_{\pi_{\varepsilon, f_{n, 1}}}(N)=K_{\varepsilon, n}, \\
M_{p, \varepsilon, \Gamma_{g_{n}}} & =\operatorname{Inv}_{\pi_{\varepsilon, n}}\left(V_{p} \cap X_{n}\right)=\operatorname{Inv}_{\pi_{\varepsilon, f_{n, 1}}}\left(V_{p}\right)=M_{p, \varepsilon, n}, \quad p \in P .
\end{aligned}
$$

Hence

$$
\left.\left.\mathcal{H}\left(\pi_{\varepsilon, n}, K_{\varepsilon, n},\left(M_{p, \varepsilon, n}\right)_{p \in P}\right) \cong \mathcal{H}\left(\pi_{n}^{\prime}, K_{n}^{\prime},\left(M_{p, n}^{\prime}\right)_{p \in P}\right), \quad \varepsilon \in\right] 0, \widetilde{\varepsilon}_{0}\right]
$$

Define $\varepsilon_{0}:=\min \left\{\varepsilon^{\prime}, \widetilde{\varepsilon}_{0}\right\}$, where $\left.\varepsilon^{\prime} \in\right] 0, \infty[$ is as in Lemma 4.8, and let $\left.\varepsilon \in] 0, \varepsilon_{0}\right]$. Proposition 4.11 implies that

$$
\mathcal{H}\left(\pi_{\varepsilon, n}, K_{\varepsilon, n},\left(M_{p, \varepsilon, n}\right)_{p \in P}\right) \cong \mathcal{H}\left(\pi_{\varepsilon}, K_{\varepsilon},\left(M_{p, \varepsilon}\right)_{p \in P}\right) .
$$

Now (4.10)-(4.12) imply that

$$
\left.\left.\mathcal{H}\left(\pi_{\varepsilon}, K_{\varepsilon},\left(M_{p, \varepsilon}\right)_{p \in P}\right) \cong \mathcal{H}\left(\pi^{\prime}, K^{\prime},\left(M_{p}^{\prime}\right)_{p \in P}\right), \quad \varepsilon \in\right] 0, \varepsilon_{0}\right]
$$

and the proof is complete.

\section{References}

[1] M. C. Carbinatto and K. P. Rybakowski, Morse decompositions in the absence of uniqueness, Topol. Methods Nonlinear Anal. 18 (2001), 205-242. 
[2] M. C. Carbinatto and K. P. Rybakowski, On a general Conley index continuation principle for singular perturbation problems, Ergodic Theory Dynam. Systems 22 (2002), 729-755.

[3] -, - On convergence, admissibility and attractors for damped wave equations on squeezed domains, Proc. Roy. Soc. Edinburgh Sect. A 132 (2002), 765-791.

[4] - - - Morse decompositions in the absence of uniqueness, II, Topol. Methods Nonlinear Anal. 22 (2003), 17-53.

[5] - - - Nested sequences of index filtrations and continuation of the connection matrix, J. Differential Equations 207 (2004), 458-488.

[6] - - - Homology index braids in infinite-dimensional Conley index theory, Topol. Methods Nonlinear Anal. 26 (2005), 35-74.

[7] - - - Continuation of the connection matrix in singular perturbation problems, Ergodic Theory Dynam. Systems 26 (2006), 1021-1059.

[8] - - - The suspension isomorphism for homology index braids, Topol. Methods Nonlinear Anal. 28 (2006), 199-233.

[9] R. D. Franzosa, Index filtrations and the homology index braid for partially ordered Morse decompositions, Trans. Amer. Math. Soc. 298 (1986), 193-213.

[10] - , The continuation theory for Morse decompositions and connection matrices, ibid. 310 (1988), 781-803.

[11] R. D. Franzosa and K. Mischaikow, The connection matrix theory for semiflows on (not necessarily locally compact) metric spaces, J. Differential Equations 71 (1988), $270-287$.

[12] J. Hale and G. Raugel, Upper semicontinuity of the attractor for a singularly perturbed hyperbolic equation, ibid. 73 (1988), 197-214.

[13] D. Henry, Geometric Theory of Semilinear Parabolic Equations, Springer, Berlin, 1981.

[14] K. P. Rybakowski, On the homotopy index for infinite-dimensional semiflows, Trans. Amer. Math. Soc. 269 (1982), 351-382.

[15] - The Morse index, repeller-attractor pairs and the connection index for semiflows on noncompact spaces, J. Differential Equations 47 (1983), 66-98.

[16] - The Homotopy Index and Partial Differential Equations, Springer, Berlin, 1987.

[17] - Conley index continuation for singularly perturbed hyperbolic equations, Topol. Methods Nonlinear Anal. 22 (2003), 203-244.

[18] —, The suspension isomorphism for cohomology index braids, ibid. 29 (2007), 1-28.

Departamento de Matemática, ICMC-USP

Caixa Postal 668

13.560-970 São Carlos SP, Brazil

E-mail: mdccarbi@icmc.usp.br
Institut für Mathematik Universität Rostock Universitätsplatz 1

18055 Rostock, Germany

E-mail: krzysztof.rybakowski@mathematik.uni-rostock.de

Received 19 April 2006;

in revised form 28 August 2007 